\title{
Optimization of Low-Thrust Limited-Power Trajectories in a Noncentral Gravity Field-Transfers between Orbits with Small Eccentricities
}

\author{
Sandro da Silva Fernandes \\ Departamento de Matemática, Instituto Tecnológico de Aeronáutica, 12228-900 São José dos Campos, \\ SP, Brazil \\ Correspondence should be addressed to Sandro da Silva Fernandes, sandro@ief.ita.br \\ Received 12 October 2008; Revised 14 February 2009; Accepted 15 March 2009 \\ Recommended by Dane Quinn
}

Numerical and first-order analytical results are presented for optimal low-thrust limited-power trajectories in a gravity field that includes the second zonal harmonic $J_{2}$ in the gravitational potential. Only transfers between orbits with small eccentricities are considered. The optimization problem is formulated as a Mayer problem of optimal control with Cartesian elements-position and velocity vectors-as state variables. After applying the Pontryagin Maximum Principle, successive canonical transformations are performed and a suitable set of orbital elements is introduced. Hori method-a perturbation technique based on Lie series-is applied in solving the canonical system of differential equations that governs the optimal trajectories. First-order analytical solutions are presented for transfers between close orbits, and a numerical solution is obtained for transfers between arbitrary orbits by solving the two-point boundary value problem described by averaged maximum Hamiltonian, expressed in nonsingular elements, through a shooting method. A comparison between analytical and numerical results is presented for some maneuvers.

Copyright (c) 2009 Sandro da Silva Fernandes. This is an open access article distributed under the Creative Commons Attribution License, which permits unrestricted use, distribution, and reproduction in any medium, provided the original work is properly cited.

\section{Introduction}

This paper presents numerical and analytical results for optimal low-thrust limited-power transfers between orbits with small eccentricities in a noncentral gravity field that includes the second zonal harmonic $J_{2}$ in the gravitational potential. This study has been motivated by the renewed interest in the use of low-thrust propulsion systems in space missions verified in the last two decades due to the development and the successes of two space mission powered by ionic propulsion: Deep Space One and SMART 1. Several researchers have obtained numerical and sometimes analytical solutions for a number of specific initial orbits and specific thrust profiles in central or noncentral gravity field [1-26]. 
It is assumed that the thrust direction is free and the thrust magnitude is unbounded $[5,27]$, that is, there exist no constraints on control variables. It is supposed that the orbital changes caused by the thrust and the perturbations due to the oblateness of the central body have the same order of magnitude. The optimization problem is formulated as a Mayer problem of optimal control with position and velocity vectors-Cartesian elements-as state variables. After applying the Pontryagin Maximum Principle [28], successive Mathieu transformations are performed and a suitable set of orbital elements is introduced. Hori method - a perturbation technique based on Lie series [29] — is applied in solving the canonical system of differential equations that governs the optimal trajectories. For transfers between close quasicircular orbits, new set of nonsingular orbital elements is introduced and the new Hamiltonian and the generating function, determined through the algorithm of Hori method, are transformed to these new elements through Mathieu transformations. First-order analytical solutions are obtained for transfers between close quasicircular orbits, considering maneuvers between near-equatorial orbits or nonequatorial orbits. These solutions are given in terms of systems of algebraic equations involving the imposed variations of the orbital elements, the duration of the maneuvers, the effects of the oblateness of the central body, and the initial values of the adjoint variables. The study of these transfers is particularly interesting because the orbits found in practice often have a small eccentricity, and the problem of slight modifications (corrections) of these orbits is frequently met [5]. For transfers between arbitrary orbits, a slightly different set of nonsingular elements is introduced, and the two-point boundary value problem described by averaged maximum Hamiltonian, expressed in nonsingular elements, is solved through a shooting method. A comparison between analytical and numerical results is presented for some maneuvers.

\section{Optimal Space Trajectories}

A low-thrust limited-power propulsion system, or LP system, is characterized by low-thrust acceleration level and high specific impulse [5]. The ratio between the maximum thrust acceleration and the gravity acceleration on the ground, $\gamma_{\max } / g_{0}$, is between $10^{-4}$ and $10^{-2}$. For such system, the fuel consumption is described by the variable $J$ defined as

$$
J=\frac{1}{2} \int_{t_{0}}^{t} \gamma^{2} d t
$$

where $\gamma$ is the magnitude of the thrust acceleration vector $r$, used as control variable. The consumption variable $J$ is a monotonic decreasing function of the mass $m$ of space vehicle:

$$
J=P_{\max }\left(\frac{1}{m}-\frac{1}{m_{0}}\right)
$$

where $P_{\max }$ is the maximum power, and $m_{0}$ is the initial mass. The minimization of the final value of the fuel consumption $J_{f}$ is equivalent to the maximization of $m_{f}$.

The general optimization problem concerned with low-thrust limited-power transfers (no rendezvous) will be formulated as a Mayer problem of optimal control by using Cartesian elements as state variables. Consider the motion of a space vehicle $M$ powered by a limitedpower engine in a general gravity field. At time $t$, the state of the vehicle is defined by the 


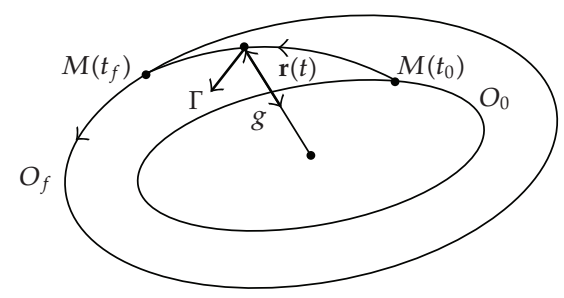

Figure 1: Geometry of the transfer problem.

position vector $\mathbf{r}(t)$, the velocity vector $\mathbf{v}(t)$, and the consumption variable $J$. The geometry of the transfer problem is illustrated in Figure 1. The control $\gamma$ is unconstrained, that is, the thrust direction is free, and the thrust magnitude is unbounded.

The optimization problem is formulated as follows: it is proposed to transfer the space vehicle $M$ from the initial state $\left(\mathbf{r}_{0}, \mathbf{v}_{0}, 0\right)$ at the initial time $t_{0}=0$ to the final state $\left(\mathbf{r}_{f}, \mathbf{v}_{f}, J_{f}\right)$ at the specified final time $t_{f}$, such that the final consumption variable $J_{f}$ is a minimum. The state equations are

$$
\frac{d \mathbf{r}}{d t}=\mathbf{v}, \quad \frac{d \mathbf{v}}{d t}=\mathbf{g}(\mathbf{r})+r, \quad \frac{d J}{d t}=\frac{1}{2} r^{2},
$$

where $\mathbf{g}(\mathbf{r})$ is the gravity acceleration. For transfers between orbits with small eccentricities, the initial and final conditions will be specified in terms of singular orbital elements introduced in next sections. It is also assumed that $t_{f}-t_{0}$ and the position of the vehicle in the initial orbit are specified.

According to the Pontryagin Maximum Principle [28], the optimal thrust acceleration $r^{*}$ must be selected from the admissible controls such that the Hamiltonian function $H$ reaches its maximum. The Hamiltonian function is formed using (2.3),

$$
H=\mathbf{p}_{r} \bullet \mathbf{v}+\mathbf{p}_{v} \bullet(\mathbf{g}(\mathbf{r})+r)+\frac{1}{2} p_{J} r^{2}
$$

where $\mathbf{p}_{r}, \mathbf{p}_{v}$, and $p_{J}$ are the adjoint variables, and dot denotes the dot product. Since the optimization problem is unconstrained, $\boldsymbol{r}^{*}$ is given by

$$
r^{*}=-\frac{\mathbf{p}_{v}}{p_{J}} .
$$

The optimal thrust acceleration $\gamma^{*}$ is modulated [5], and the optimal trajectories are governed by the maximum Hamiltonian function $H^{*}$, obtained from (2.4) and (2.5):

$$
H^{*}=\mathbf{p}_{r} \bullet \mathbf{v}+\mathbf{p}_{v} \bullet \mathbf{g}(\mathbf{r})-\frac{p_{v}{ }^{2}}{2 p_{J}}
$$


The consumption variable $J$ is ignorable, and $p_{J}$ is a first integral. From the transversality conditions, $p_{J}\left(t_{f}\right)=-1$; thus,

$$
p_{J}(t)=-1
$$

Equation (2.6) reduces to

$$
H=\mathbf{p}_{r} \bullet \mathbf{v}+\mathbf{p}_{v} \bullet \mathbf{g}(\mathbf{r})+\frac{p_{v}{ }^{2}}{2} .
$$

The consumption variable $J$ is determined by simple quadrature.

For noncentral field, the gravity acceleration is given by [30]

$$
\mathbf{g}(\mathbf{r})=-\frac{\mu}{r^{3}} \mathbf{r}+\left(\frac{\partial V_{2}}{\partial \mathbf{r}}\right)^{T}
$$

with the disturbing function $V_{2}$ defined by

$$
V_{2}=-\mu J_{2}\left(\frac{a_{e}{ }^{2}}{r^{3}}\right) P_{2}(\sin \varphi),
$$

where $\mu$ is the gravitational parameter, $a_{e}$ is the mean equatorial radius of the central body, $J_{2}$ is the coefficient for the second zonal harmonic of the potential, $\varphi$ is the latitude, and $P_{2}$ is the Legendre polynomial of order $2 .\left(\partial V_{2} / \partial \mathbf{r}\right)$ is a row vector of partial derivatives, and superscript $T$ denotes its transpose. form:

Using (2.8) and (2.9), the maximum Hamiltonian function can be put in the following

$$
H^{*}=H_{0}+H_{J_{2}}+H_{\gamma^{*}}
$$

where

$$
\begin{gathered}
H_{0}=\mathbf{p}_{r} \bullet \mathbf{v}-\mathbf{p}_{v} \bullet \frac{\mu}{r^{3}} \mathbf{r}, \\
H_{J_{2}}=\mathbf{p}_{v} \bullet\left(\frac{\partial V_{2}}{\partial \mathbf{r}}\right)^{T}, \\
H_{r_{*}}=\frac{p_{v}^{2}}{2} .
\end{gathered}
$$

$H_{0}$ is the undisturbed Hamiltonian function, $H_{J_{2}}$ and $H_{\gamma^{*}}$ are disturbing functions concerning the oblateness of the central body and the optimal thrust acceleration, respectively. $H_{J_{2}}$ and $H_{\gamma *}$ are assumed to be of the same order of magnitude. 


\section{Transformation from Cartesian Elements to a Set of Orbital Elements}

Consider the canonical system of differential equations governed by the undisturbed Hamiltonian $H_{0}$ :

$$
\begin{gathered}
\frac{d \mathbf{r}}{d t}=\mathbf{v}, \quad \frac{d \mathbf{v}}{d t}=-\frac{\mu}{r^{3}} \mathbf{r} \\
\frac{d \mathbf{p}_{\mathbf{r}}}{d t}=\frac{\mu}{r^{3}}\left(\mathbf{p}_{v}-3\left(\mathbf{p}_{v} \bullet \mathbf{e}_{\mathbf{r}}\right) \mathbf{e}_{\mathbf{r}}\right), \quad \frac{d \mathbf{p}_{v}}{d t}=-\mathbf{p}_{r},
\end{gathered}
$$

where $\mathbf{e}_{\mathbf{r}}$ is the unit vector pointing radially outward of the moving frame of reference (Figure 2). The general solution of the state equations is well known in Astrodynamics [31], and the general solution of the adjoint equations is obtained through properties of generalized canonical systems [32], as described in the appendix. Thus,

$$
\begin{aligned}
& \mathbf{r}=\frac{a\left(1-e^{2}\right)}{1+e \cos f} \mathbf{e}_{\mathbf{r}} \\
& \mathbf{v}=\sqrt{\frac{\mu}{a\left(1-e^{2}\right)}}\left[e \sin f \mathbf{e}_{\mathbf{r}}+(1+e \cos f) \mathbf{e}_{\mathbf{s}}\right], \\
& \mathbf{p}_{\mathbf{r}}=\frac{a}{r^{2}}\left\{2 a p_{a}+\left(\left(1-e^{2}\right) \cos E\right) p_{e}+\left(\frac{r}{a}\right) \frac{\sin f}{e}\left(p_{\omega}-\frac{\left(1-e^{3} \cos E\right)}{\sqrt{1-e^{2}}} p_{M}\right)\right\} \mathbf{e}_{\mathbf{r}} \\
& +\left\{\frac{\sin f}{a} p_{e}-\frac{(e+\cos f)}{a e\left(1-e^{2}\right)} p_{\omega}+\frac{\sqrt{1-e^{2}} \cos f}{a e} p_{M}\right\} \mathbf{e}_{\mathbf{s}} \\
& +\frac{1}{a \sqrt{1-e^{2}}}\left\{\left(\frac{a}{r}\right) \sin E\left[p_{I} \cos \omega+\left(\frac{p_{\Omega}}{\sin I}-p_{\omega} \cot I\right) \sin \omega\right]\right. \\
& \left.+\sqrt{1-e^{2}}\left(\frac{a}{r}\right) \cos E\left[p_{I} \sin \omega-\left(\frac{p_{\Omega}}{\sin I}-p_{\omega} \cot I\right) \cos \omega\right]\right\} \mathbf{e}_{\mathrm{w}}, \\
& \mathbf{p}_{\mathbf{v}}=\frac{1}{n a \sqrt{1-e^{2}}}\left\{\left\{2 a e \sin f p_{a}+\left(\left(1-e^{2}\right) \sin f\right) p_{e}-\frac{\left(1-e^{2}\right) \cos f}{e} p_{\omega}\right.\right. \\
& \left.+\frac{\left(1-e^{2}\right)^{3 / 2}}{e}\left(\cos f-\frac{2 e}{1+e \cos f}\right) p_{M}\right\} \mathbf{e}_{\mathrm{r}} \\
& +\left\{2 a\left(1-e^{2}\right)\left(\frac{a}{r}\right) p_{a}+\left(1-e^{2}\right)(\cos f+\cos E) p_{e}\right. \\
& \left.+\frac{\left(1-e^{2}\right) \sin f}{e}\left(1+\frac{1}{1+e \cos f}\right)\left(p_{\omega}-\sqrt{1-e^{2}} p_{M}\right)\right\} \mathbf{e}_{\mathbf{s}} \\
& \left.+\left\{\left(\frac{r}{a}\right) \cos (\omega+f) p_{I}+\left(\frac{r}{a}\right) \sin (\omega+f)\left(\frac{p_{\Omega}}{\sin I}-p_{\omega} \cot I\right)\right\} \mathbf{e}_{\mathrm{w}}\right\},
\end{aligned}
$$




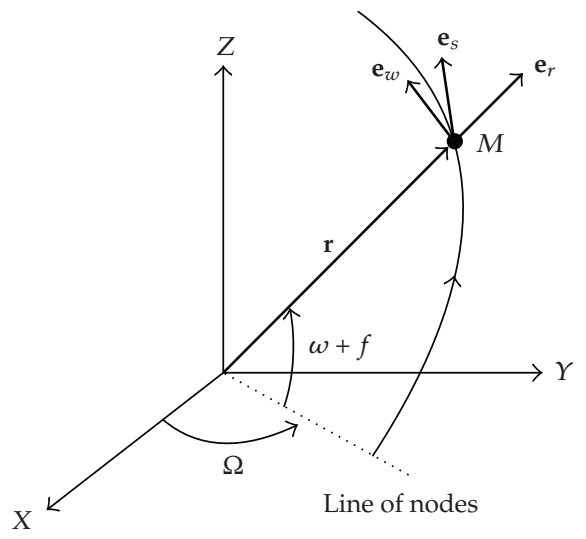

Figure 2: Frames of reference.

where $\mathbf{e}_{\mathrm{s}}$ and $\mathbf{e}_{\mathrm{w}}$ are unit vectors along circumferential and normal directions of the moving frame of reference, respectively; $a$ is the semimajor axis, $e$ is the eccentricity, $I$ is the inclination of orbital plane, $\Omega$ is the longitude of the ascending node, $\omega$ is the argument of pericenter, $f$ is the true anomaly, $E$ is the eccentric anomaly, $M$ is the mean anomaly, $n=\sqrt{\mu / a^{3}}$ is the mean motion, and $(r / a),(r / a) \sin f$, and so forth are functions of the elliptic motion which can be expressed explicitly in terms of the eccentricity and the mean anomaly through Lagrange series [31]. The true, eccentric and mean anomalies are related through the equations:

$$
\begin{aligned}
\tan \frac{f}{2} & =\sqrt{\frac{1+e}{1-e}} \tan \frac{E}{2} \\
M & =E-e \sin E .
\end{aligned}
$$

The unit vectors $\mathbf{e}_{\mathbf{r}}, \mathbf{e}_{\mathrm{s}}$, and $\mathbf{e}_{\mathrm{w}}$ of the moving frame of reference are written in the fixed frame of reference as

$$
\begin{aligned}
\mathbf{e}_{\mathbf{r}}= & (\cos \Omega \cos (\omega+f)-\sin \Omega \sin (\omega+f) \cos I) \mathbf{i} \\
& +(\sin \Omega \cos (\omega+f)+\cos \Omega \sin (\omega+f) \cos I) \mathbf{j}+\sin (\omega+f) \sin I \mathbf{k}, \\
\mathbf{e}_{\mathbf{s}}= & -(\cos \Omega \sin (\omega+f)+\sin \Omega \cos (\omega+f) \cos I) \mathbf{i} \\
& +(-\sin \Omega \sin (\omega+f)+\cos \Omega \cos (\omega+f) \cos I) \mathbf{j}+\cos (\omega+f) \sin I \mathbf{k}, \\
\mathbf{e}_{\mathbf{w}}= & \sin \Omega \sin I \mathbf{i}-\cos \Omega \sin I \mathbf{j}+\cos I \mathbf{k} .
\end{aligned}
$$

Equations (3.2)-(3.5) define a Mathieu transformation between the Cartesian elements $\left(\mathbf{r}, \mathbf{v}, \mathbf{p}_{\mathbf{r}}, \mathbf{p}_{\mathbf{v}}\right)$ and the orbital ones $\left(a, e, I, \Omega, \omega, M, p_{a}, p_{e}, p_{I}, p_{\Omega}, p_{\omega}, p_{M}\right)$. Since the Hamiltonian function is invariant with respect to this canonical transformation, it follows, from (2.11) through (3.5), that

$$
H^{*}=H_{0}+H_{J_{2}}+H_{\gamma^{*}}
$$


where

$$
\begin{aligned}
& H_{0}=n p_{M} \\
& H_{J_{2}}=\frac{2}{n a} \frac{\partial V_{2}^{\prime}}{\partial M} p_{a}+\frac{\sqrt{1-e^{2}}}{n a^{2} e}\left[-\frac{\partial V_{2}^{\prime}}{\partial \omega}+\sqrt{1-e^{2}} \frac{\partial V_{2}^{\prime}}{\partial M}\right] p_{e} \\
& +\frac{1}{n a^{2} \sqrt{1-e^{2}} \sin I}\left[-\frac{\partial V_{2}^{\prime}}{\partial \Omega}+\cos I \frac{\partial V_{2}^{\prime}}{\partial \omega}\right] p_{I} \\
& +\frac{1}{n a^{2} \sqrt{1-e^{2}} \sin I} \frac{\partial V_{2}^{\prime}}{\partial I} p_{\Omega}+\frac{\sqrt{1-e^{2}}}{n a^{2} e}\left[\frac{\partial V_{2}^{\prime}}{\partial e}-\frac{e \cot I}{\left(1-e^{2}\right)} \frac{\partial V_{2}^{\prime}}{\partial I}\right] p_{\omega} \\
& \left.+\frac{1}{n a}\left[-2 \frac{\partial V_{2}^{\prime}}{\partial a}-\frac{\left(1-e^{2}\right)}{a e} \frac{\partial V_{2}^{\prime}}{\partial e}\right] p_{\mathrm{M}}\right\} \\
& H_{\gamma}^{*}=\frac{1}{2 n^{2} a^{2}\left(1-e^{2}\right)}\left\{\frac{1}{2}(1-\cos 2 f)\left[2 a e p_{a}+\left(1-e^{2}\right) p_{e}\right]^{2}\right. \\
& +2\left(1-e^{2}\right) \sin 2 f\left[-a p_{a} p_{\omega}-\frac{\left(1-e^{2}\right)}{2 e} p_{e} p_{\omega}\right] \\
& +4\left(1-e^{2}\right)^{3 / 2} \sin f\left(\frac{-2 e}{1+e \cos f}+\cos f\right)\left[a p_{a} p_{M}+\frac{\left(1-e^{2}\right)}{2 e} p_{e} p_{M}\right] \\
& +\frac{\left(1-e^{2}\right)^{2}}{2 e^{2}}(1+\cos 2 f) p_{\omega}^{2} \\
& -\frac{2\left(1-e^{2}\right)^{5 / 2}}{e^{2}}\left(\frac{-2 e}{1+e \cos f}+\cos f\right) \cos f p_{\omega} p_{M} \\
& +\frac{\left(1-e^{2}\right)^{3}}{e^{2}}\left(\frac{-2 e}{1+e \cos f}+\cos f\right)^{2} p_{M}^{2}+4 a^{2}\left(1-e^{2}\right)^{2}\left(\frac{a}{r}\right)^{2} p_{a}^{2} \\
& +4 a\left(1-e^{2}\right)^{2}\left(\frac{a}{r}\right)(\cos E+\cos f) p_{a} p_{e} \\
& +\left(1-e^{2}\right)^{2}(\cos E+\cos f)^{2} p_{e}{ }^{2} \\
& +\frac{4 a\left(1-e^{2}\right)^{2}}{e}\left(\frac{a}{r}\right) \sin f\left(1+\frac{1}{1+e \cos f}\right) \\
& \times\left[p_{a} p_{\omega}-\left(1-e^{2}\right)^{1 / 2} p_{a} p_{M}\right] \\
& +\frac{2\left(1-e^{2}\right)^{2}}{e}(\cos E+\cos f)\left(1+\frac{1}{1+e \cos f}\right) \\
& \times \sin f\left[p_{e} p_{\omega}-\sqrt{1-e^{2}} p_{e} p_{M}\right]
\end{aligned}
$$




$$
\begin{aligned}
& +\left[\frac{\left(1-e^{2}\right)}{e}\left(1+\frac{1}{1+e \cos f}\right) \sin f\left[p_{\omega}-\sqrt{1-e^{2}} p_{M}\right]\right]^{2} \\
& +\frac{1}{2}\left(\frac{r}{a}\right)^{2}\left[p_{I}^{2}+\left(\frac{p_{\Omega}}{\sin I}-p_{\omega} \cot I\right)^{2}\right] \\
& +\frac{1}{2}\left(\frac{r}{a}\right)^{2} \cos 2(\omega+f)\left[p_{I}^{2}-\left(\frac{p_{\Omega}}{\sin I}-p_{\omega} \cot I\right)^{2}\right] \\
& \left.+\left(\frac{r}{a}\right)^{2} \sin 2(\omega+f) p_{I}\left(\frac{p_{\Omega}}{\sin I}-p_{\omega} \cot I\right)\right\}
\end{aligned}
$$

with the disturbing function $V_{2}^{\prime}$ given by

$$
V_{2}^{\prime}=\frac{\mu}{a}\left(\frac{a_{e}}{a}\right)^{2} J_{2}\left\{\left(\frac{a}{r}\right)^{3}\left[\frac{1}{2}-\frac{3}{4} \sin ^{2} I\right]+\frac{3}{4}\left(\frac{a}{r}\right)^{3} \sin ^{2} I \cos 2(\omega+f)\right\}
$$

The new Hamiltonian function $H^{*}$, defined by (3.8)-(3.10), describes the optimal lowthrust limited-power trajectories in a noncentral gravity field which includes the perturbative effects of the second zonal harmonic of the gravitational potential.

\section{Averaged Maximum Hamiltonian for Optimal Transfers}

In order to eliminate the short periodic terms from the maximum Hamiltonian function $H^{*}$, Hori method [29] is applied. It is assumed that $H_{0}$ is of zero-order and $H_{\gamma}^{*}$ is of the first-order in a small parameter associated to the magnitude of the thrust acceleration, and $H_{J_{2}}$ has the same order of $H_{r}^{*}$.

Consider an infinitesimal canonical transformation:

$$
\left(a, e, I, \Omega, \omega, M, p_{a}, p_{e}, p_{I}, p_{\Omega}, p_{\omega}, p_{M}\right) \longrightarrow\left(a^{\prime}, e^{\prime}, I^{\prime}, \Omega^{\prime}, \omega^{\prime}, M^{\prime}, p_{a}^{\prime}, p_{e}^{\prime}, p_{I^{\prime}}^{\prime} p_{\Omega^{\prime}}^{\prime} p_{\omega}^{\prime}, p_{M}^{\prime}\right)
$$

The new variables are designated by the prime. According to the algorithm of Hori method [29], at order 0,

$$
F_{0}=n^{\prime} p_{M}^{\prime}
$$

$F_{0}$ denotes the new undisturbed Hamiltonian. Now, consider the canonical system described by $F_{0}$ :

$$
\begin{aligned}
& \frac{d a^{\prime}}{d t}=0, \quad \frac{d e^{\prime}}{d t}=0, \quad \frac{d I^{\prime}}{d t}=0, \\
& \frac{d \Omega^{\prime}}{d t}=0, \quad \frac{d \omega^{\prime}}{d t}=0, \quad \frac{d M^{\prime}}{d t}=n^{\prime},
\end{aligned}
$$


Mathematical Problems in Engineering

$$
\begin{aligned}
& \frac{d p_{a}^{\prime}}{d t}=\frac{3}{2} \frac{n^{\prime}}{a^{\prime}} p_{M^{\prime}}^{\prime} \quad \frac{d p_{e}^{\prime}}{d t}=0, \quad \frac{d p_{I}^{\prime}}{d t}=0, \\
& \frac{d p_{\Omega}^{\prime}}{d t}=0, \quad \frac{d p_{\omega}^{\prime}}{d t}=0, \quad \frac{d p_{M}^{\prime}}{d t}=0,
\end{aligned}
$$

general solution of which is given by

$$
\begin{gathered}
a^{\prime}=a_{0}^{\prime}, \quad e^{\prime}=e_{0}^{\prime}, \quad I^{\prime}=I_{0}^{\prime}, \\
\Omega^{\prime}=\Omega_{0}^{\prime}, \quad \omega^{\prime}=\omega_{0}^{\prime}, \quad M^{\prime}=M_{0}^{\prime}+n^{\prime}\left(t-t_{0}\right), \\
p_{a}^{\prime}=p_{a_{0}}^{\prime}+\frac{3}{2} \frac{n^{\prime}\left(t-t_{0}\right)}{a^{\prime}} p_{M^{\prime}}^{\prime} \quad p_{e}^{\prime}=p_{e_{0}}^{\prime} \quad p_{I}^{\prime}=p_{I_{0},}^{\prime} \\
p_{\Omega}^{\prime}=p_{\Omega_{0}}^{\prime} \quad p_{\omega}^{\prime}=p_{\omega_{0},}^{\prime} \quad p_{M}^{\prime}=p_{M_{0}}^{\prime} .
\end{gathered}
$$

The subscript 0 denotes the constants of integration.

Introducing the general solution defined above into the equation of order 1 of the algorithm of Hori method, it reduces to

$$
\frac{\partial S_{1}}{\partial t}=H_{J_{2}}+H_{r^{*}}-F_{1}
$$

According to [29], the mean value of $H_{J_{2}}+H_{\gamma^{*}}$ must be calculated, and, $S_{1}$ is obtained through integration of the remaining part. $F_{1}$ and $S_{1}$ are given by the following equations:

$$
\begin{aligned}
F_{1}= & -\frac{3}{2} n^{\prime} J_{2}\left(\frac{a_{e}}{a^{\prime}}\right)^{2}\left(1-e^{\prime 2}\right)^{-2}\left\{\cos I^{\prime} p_{\Omega}^{\prime}+\frac{1}{2}\left(1-5 \cos ^{2} I^{\prime}\right) p_{\omega}^{\prime}\right\} \\
+ & \frac{a^{\prime}}{2 \mu}\left\{4 a^{\prime 2} p_{a}^{\prime 2}+\frac{5}{2}\left(1-e^{\prime 2}\right) p_{e}^{\prime 2}+\frac{\left(5-4 e^{\prime 2}\right)}{2 e^{\prime 2}} p_{\omega}^{\prime 2}\right. \\
& +\frac{p_{I}^{\prime 2}}{2\left(1-e^{\prime 2}\right)}\left[\left(1+\frac{3}{2} e^{\prime 2}\right)+\frac{5}{2} e^{\prime 2} \cos 2 \omega^{\prime}\right]+\frac{5 e^{\prime 2} \sin 2 \omega^{\prime}}{2\left(1-e^{\prime 2}\right)} p_{I}^{\prime}\left(\frac{p_{\Omega}^{\prime}}{\sin I^{\prime}}-\cot I^{\prime} p_{\omega}^{\prime}\right) \\
& \left.+\frac{1}{2\left(1-e^{\prime 2}\right)}\left(\frac{p_{\Omega}^{\prime}}{\sin I^{\prime}}-\cot I^{\prime} p_{\omega}^{\prime}\right)^{2}\left[\left(1+\frac{3}{2} e^{\prime 2}\right)-\frac{5}{2} e^{\prime 2} \cos 2 \omega^{\prime}\right]\right\},
\end{aligned}
$$




$$
\begin{aligned}
& S_{1}=J_{2}\left(\frac{a_{e}}{a^{\prime}}\right)^{2}\left\{\left\{\left(1-\frac{3}{2} \sin ^{2} I^{\prime}\right)\left[\left(\frac{a^{\prime}}{r^{\prime}}\right)^{3}-\left(1-e^{\prime 2}\right)^{-3 / 2}\right]+\frac{3}{2} \sin ^{2} I^{\prime}\left(\frac{a^{\prime}}{r^{\prime}}\right)^{3} \cos 2\left(\omega^{\prime}+f^{\prime}\right)\right\} a^{\prime} p_{a}^{\prime}\right. \\
& +\left\{\frac{3}{2} \frac{\sin ^{2} I^{\prime}}{e^{\prime}\left(1-e^{\prime 2}\right)}\left[-\frac{1}{2} \cos 2\left(\omega^{\prime}+f^{\prime}\right)-\frac{e^{\prime}}{2}\left(\cos \left(2 \omega^{\prime}+f^{\prime}\right)+\frac{1}{3} \cos \left(2 \omega^{\prime}+3 f^{\prime}\right)\right)\right]\right. \\
& +\frac{\left(1-e^{\prime 2}\right)}{e^{\prime}}\left[\left(\frac{1}{2}-\frac{3}{4} \sin ^{2} I^{\prime}\right)\left[\left(\frac{a^{\prime}}{r^{\prime}}\right)^{3}-\left(1-e^{\prime 2}\right)^{-3 / 2}\right]\right. \\
& \left.+\frac{3}{4} \sin ^{2} I^{\prime}\left(\frac{a^{\prime}}{r^{\prime}}\right)^{3} \cos 2\left(\omega^{\prime}+f^{\prime}\right)\right\} p_{e}^{\prime} \\
& +\left\{\frac{3}{4} \frac{\sin 2 I^{\prime}}{\left(1-e^{\prime 2}\right)^{2}}\left[\frac{1}{2} \cos 2\left(\omega^{\prime}+f^{\prime}\right)+\frac{e^{\prime}}{2}\left(\cos \left(2 \omega^{\prime}+f^{\prime}\right)+\frac{1}{3} \cos \left(2 \omega^{\prime}+3 f^{\prime}\right)\right)\right]\right\} p_{I}^{\prime} \\
& +\left\{\frac { 3 } { 2 } \frac { \operatorname { c o s } I ^ { \prime } } { ( 1 - e ^ { \prime 2 } ) ^ { 2 } } \left[-\left(f^{\prime}-M^{\prime}+e^{\prime} \sin f^{\prime}\right)+\frac{1}{2} \sin 2\left(\omega^{\prime}+f^{\prime}\right)\right.\right. \\
& \left.\left.+\frac{e^{\prime}}{2}\left(\sin \left(2 \omega^{\prime}+f^{\prime}\right)+\frac{1}{3} \sin \left(2 \omega^{\prime}+3 f^{\prime}\right)\right)\right]\right\} p_{\Omega}^{\prime} \\
& +\left\{\frac{3}{4} \frac{\left(5 \cos ^{2} I^{\prime}-1\right)}{\left(1-e^{\prime 2}\right)^{2}}\left(f^{\prime}-M^{\prime}+e^{\prime} \sin f^{\prime}\right)\right. \\
& +\frac{1}{4} \frac{\left(3 \cos ^{2} I^{\prime}-1\right)}{e^{\prime}\left(1-e^{\prime 2}\right)}\left[\left(\frac{a^{\prime}}{r^{\prime}}\right)^{2}\left(1-e^{\prime 2}\right)+\left(\frac{a^{\prime}}{r^{\prime}}\right)+1\right] \sin f^{\prime} \\
& +\frac{3}{8} \frac{\sin ^{2} I^{\prime}}{e^{\prime}\left(1-e^{\prime 2}\right)}\left[\left[-\left(\frac{a^{\prime}}{r^{\prime}}\right)^{2}\left(1-e^{\prime 2}\right)-\left(\frac{a^{\prime}}{r^{\prime}}\right)+1\right] \sin \left(2 \omega^{\prime}+f^{\prime}\right)\right. \\
& \left.+\left[\left(\frac{a^{\prime}}{r^{\prime}}\right)^{2}\left(1-e^{\prime 2}\right)+\left(\frac{a^{\prime}}{r^{\prime}}\right)+\frac{1}{3}\right] \sin \left(2 \omega^{\prime}+3 f^{\prime}\right)\right] \\
& +\frac{3}{8} \frac{\left(3-5 \cos ^{2} I^{\prime}\right)}{\left(1-e^{\prime 2}\right)^{2}}\left[\sin 2\left(\omega^{\prime}+f^{\prime}\right)\right. \\
& \left.\left.\left.+e^{\prime}\left(\sin \left(2 \omega^{\prime}+f^{\prime}\right)+\frac{1}{3} \sin \left(2 \omega^{\prime}+3 f^{\prime}\right)\right)\right]\right\} p_{\omega}^{\prime}\right\} \\
& +\frac{1}{2} \sqrt{\frac{a^{\prime 5}}{\mu^{3}}}\left\{8 e^{\prime} \sin E^{\prime} a^{\prime 2} p_{a}^{\prime 2}+8\left(1-e^{\prime 2}\right) \sin E^{\prime} a^{\prime} p_{a}^{\prime} p_{e}^{\prime}-\frac{8 \sqrt{1-e^{\prime 2}}}{e^{\prime}} \cos E^{\prime} p_{a}^{\prime} p_{\omega}^{\prime}\right. \\
& +\left(1-e^{\prime 2}\right)\left[-\frac{5}{4} e^{\prime} \sin E^{\prime}+\frac{3}{4} \sin 2 E^{\prime}-\frac{1}{12} e^{\prime} \sin 3 E^{\prime}\right] p_{e}^{\prime 2} \\
& +\frac{\sqrt{1-e^{\prime 2}}}{e^{\prime}}\left[\frac{5}{2} e^{\prime} \cos E^{\prime}-\frac{1}{2}\left(3-e^{\prime 2}\right) \cos 2 E^{\prime}+\frac{1}{6} e^{\prime} \cos 3 E^{\prime}\right] p_{e}^{\prime} p_{\omega}^{\prime}
\end{aligned}
$$




$$
\begin{aligned}
& +\left(1-e^{\prime 2}\right)^{-1}\left[p_{I}^{\prime 2}+\left(\frac{p_{\Omega}^{\prime}}{\sin I^{\prime}}-p_{\omega}^{\prime} \cot I^{\prime}\right)^{2}\right] \\
& \times\left[\left(-e^{\prime}+\frac{3}{8} e^{\prime 3}\right) \sin E^{\prime}+\frac{3}{8} e^{\prime 2} \sin 2 E^{\prime}-\frac{1}{24} e^{\prime 3} \sin 3 E^{\prime}\right]+\left(1-e^{\prime 2}\right)^{-1} \\
& \times\left[p_{I}^{\prime 2} \cos 2 \omega^{\prime}+2 p_{I}^{\prime}\left(\frac{p_{\Omega}^{\prime}}{\sin I^{\prime}}-p_{\omega}^{\prime} \cot I^{\prime}\right) \sin 2 \omega^{\prime}-\left(\frac{p_{\Omega}^{\prime}}{\sin I^{\prime}}-p_{\omega}^{\prime} \cot I^{\prime}\right)^{2} \cos 2 \omega^{\prime}\right] \\
& \times\left[\left(-\frac{5}{4} e^{\prime}+\frac{5}{8} e^{\prime 3}\right) \sin E^{\prime}+\left(\frac{1}{4}+\frac{1}{8} e^{\prime 2}\right) \sin 2 E^{\prime}+\left(-\frac{1}{12} e^{\prime}+\frac{1}{24} e^{\prime 3}\right) \sin 3 E^{\prime}\right] \\
& +\left(1-e^{\prime 2}\right)^{-1 / 2}\left[-p_{I}^{\prime 2} \sin 2 \omega^{\prime}+2 p_{I}^{\prime}\left(\frac{p_{\Omega}^{\prime}}{\sin I^{\prime}}-p_{\omega}^{\prime} \cot I^{\prime}\right) \cos 2 \omega^{\prime}\right. \\
& \left.+\left(\frac{p_{\Omega}^{\prime}}{\sin I^{\prime}}-p_{\omega}^{\prime} \cot I^{\prime}\right)^{2} \sin 2 \omega^{\prime}\right] \\
& \times\left[\frac{5}{4} e^{\prime} \cos E^{\prime}-\left(\frac{1}{4}+\frac{1}{4} e^{\prime 2}\right) \cos 2 E^{\prime}+\frac{1}{12} e^{\prime} \cos 3 E^{\prime}\right] \\
& \left.+\frac{p_{\omega}^{\prime 2}}{e^{\prime 2}}\left[\left(\frac{5}{4} e^{\prime}-e^{\prime 3}\right) \sin E^{\prime}+\left(-\frac{3}{4}+\frac{1}{2} e^{\prime 2}\right) \sin 2 E^{\prime}+\frac{1}{12} e^{\prime} \sin 3 E^{\prime}\right]\right\} .
\end{aligned}
$$

Terms factored by $p_{M}^{\prime}$ have been omitted in equations above, since only transfers (no rendezvous) are considered [2].

It should be noted that the averaged maximum Hamiltonian and the generating function become singular for circular and/or equatorial orbits. In order to avoid these singularities, suitable sets of nonsingular elements will be introduced in the next sections.

\section{Optimal Transfers between Close Quasicircular Orbits}

In this section, approximate first-order analytical solutions will be obtained for transfers between close quasicircular orbits.

\subsection{Transfers between Close Quasicircular Nonequatorial Orbits}

Consider the Mathieu transformation [33] defined by

$$
\begin{gathered}
a^{\prime \prime}=a^{\prime}, \quad h^{\prime \prime}=e^{\prime} \cos \omega^{\prime}, \quad \begin{array}{l}
k^{\prime \prime}=e^{\prime} \sin \omega^{\prime}, \\
I^{\prime \prime}=I^{\prime}, \quad \Omega^{\prime \prime}=\Omega^{\prime}, \quad \ell^{\prime \prime}=M^{\prime}+\omega^{\prime},
\end{array} \\
p_{a}^{\prime \prime}=p_{a}^{\prime} \quad p_{h}^{\prime \prime}=p_{e}^{\prime} \cos \omega^{\prime}-\left(\frac{p_{\omega}^{\prime}-p_{M}^{\prime}}{e^{\prime}}\right) \sin \omega^{\prime}, \quad p_{k}^{\prime \prime}=p_{e}^{\prime} \sin \omega^{\prime}+\left(\frac{p_{\omega}^{\prime}-p_{M}^{\prime}}{e^{\prime}}\right) \cos \omega^{\prime}, \\
p_{I}^{\prime \prime}=p_{I^{\prime}}^{\prime} \quad p_{\Omega}^{\prime \prime}=p_{\Omega^{\prime}}^{\prime} \quad p_{\ell}^{\prime \prime}=p_{M}^{\prime} .
\end{gathered}
$$


The new set of canonical variables, designated by the double prime, is nonsingular for circular nonequatorial orbits.

Introducing (5.2) into (4.6) and using the expansions of the elliptic motion in terms of eccentricity and mean anomaly [31], one finds that the new averaged maximum Hamiltonian and the generating function are written up to the zeroth order in eccentricity as

$$
\begin{gathered}
F_{1}=-\frac{3}{2} n J_{2}\left(\frac{a_{e}}{a}\right)^{2} \cos I p_{\Omega}+\frac{a}{2 \mu}\left\{4 a^{2} p_{a}^{2}+\frac{5}{2}\left(p_{h}^{2}+p_{k}^{2}\right)+\frac{1}{2}\left(p_{I}^{2}+\frac{p_{\Omega}^{2}}{\sin ^{2} I}\right)\right\} \\
S_{1}=\frac{3}{2} J_{2}\left(\frac{a_{e}}{a}\right)^{2}\left\{a p_{a} \sin ^{2} I \cos 2 \ell+p_{h}\left[\cos \ell+\frac{1}{4} \sin ^{2} I\left(-5 \cos \ell+\frac{7}{3} \cos 3 \ell\right)\right]\right. \\
+p_{k}\left[\sin \ell+\frac{1}{4} \sin ^{2} I\left(-7 \sin \ell+\frac{7}{3} \sin 3 \ell\right)\right] \\
\left.+\frac{1}{4} p_{I} \sin 2 I \cos 2 \ell+\frac{1}{2} p_{\Omega} \cos I \sin 2 \ell\right\} \\
+\frac{1}{2} \sqrt{\frac{a^{5}}{\mu^{3}}}\left\{8 a p_{a}\left[p_{h} \sin \ell-p_{k} \cos \ell\right]-\frac{1}{2}\left[3 p_{h} p_{k}+\frac{p_{\Omega}}{\sin I} p_{I}\right] \cos 2 \ell\right. \\
\left.+\frac{1}{4}\left[3\left(p_{h}^{2}-p_{k}^{2}\right)+p_{I}^{2}-\frac{p_{\Omega}^{2}}{\sin ^{2} I}\right] \sin 2 \ell\right\} .
\end{gathered}
$$

Double prime is omitted to simplify the notation.

For transfers between close orbits, $F_{1}$ and $S_{1}$ can be linearized around a suitable reference orbit, and an approximate first-order analytical solution can be determined. This solution is given by

$$
\Delta x=A p_{0}+B
$$

where $\Delta x=\left[\Delta \alpha \Delta h \Delta k \Delta I \Delta \Omega^{\prime}\right]^{T}, p_{0}$ is the $5 \times 1$ vector of initial value of the adjoint variables, $A$ is a $5 \times 5$ symmetric matrix concerning the optimal thrust acceleration and $B$ is a $5 \times 1$ vector containing the perturbative effects of the oblateness of the central body. The variables $\alpha=a / \bar{a}$ and $p_{\alpha}=\bar{a} p_{a}$ are introduced to make the adjoint and the state vectors dimensionless, and the variables $\Omega^{\prime}=\Omega \sin \bar{I}$ and $p_{\Omega^{\prime}}=p_{\Omega} / \sin \bar{I}$ are introduced to simplify the matrix $A$. The adjoint vector is constant. The matrix $A$ and the vector $B$ are given by

$$
A=\left[\begin{array}{ccccc}
a_{\alpha \alpha} & a_{\alpha h} & a_{\alpha k} & 0 & 0 \\
a_{h \alpha} & a_{h h} & a_{h k} & 0 & 0 \\
a_{k \alpha} & a_{k h} & a_{k k} & 0 & 0 \\
0 & 0 & 0 & a_{I I} & a_{I \Omega^{\prime}} \\
0 & 0 & 0 & a_{\Omega^{\prime} I} & a_{\Omega^{\prime} \Omega^{\prime}}
\end{array}\right],
$$


Mathematical Problems in Engineering

$$
B=\left[\begin{array}{c}
b_{\alpha} \\
b_{h} \\
b_{k} \\
b_{I} \\
b_{\Omega^{\prime}}
\end{array}\right],
$$

where

$$
\begin{aligned}
& a_{\alpha \alpha}=\left.4 \sqrt{\frac{\bar{a}^{5}}{\mu^{3}}} \bar{\ell}\right|_{\bar{\ell}_{0}} ^{\bar{\ell}_{f}}, \\
& a_{\alpha h}=a_{h \alpha}=\left.4 \sqrt{\frac{\overline{\bar{a}^{5}}}{\mu^{3}}} \sin \bar{\ell}\right|_{\bar{\ell}_{0}} ^{\bar{\ell}_{f}}, \\
& a_{\alpha k}=a_{k \alpha}=-\left.4 \sqrt{\frac{\bar{a}^{5}}{\mu^{3}}} \cos \bar{\ell}\right|_{\bar{\ell}_{0}} ^{\bar{\ell}_{f}}, \\
& a_{h h}=\left.\sqrt{\frac{\bar{a}^{5}}{\mu^{3}}}\left[\frac{5}{2} \bar{\ell}+\frac{3}{4} \sin 2 \bar{\ell}\right]\right|_{\bar{\ell}_{0}} ^{\bar{\ell}_{f}}, \\
& a_{h k}=a_{k h}=-\left.\frac{3}{4} \sqrt{\frac{\bar{a}^{5}}{\mu^{3}}} \cos 2 \bar{\ell}\right|_{\bar{\ell}_{0}} ^{\bar{\ell}_{f}}, \\
& a_{k k}=\left.\sqrt{\frac{\bar{a}^{5}}{\mu^{3}}}\left[\frac{5}{2} \bar{\ell}-\frac{3}{4} \sin 2 \bar{\ell}\right]\right|_{\bar{\ell}_{0}} ^{\bar{\ell}_{f}}, \\
& a_{I I}=\left.\sqrt{\frac{\bar{a}^{5}}{\mu^{3}}}\left[\frac{1}{2} \bar{\ell}+\frac{1}{4} \sin 2 \bar{\ell}\right]\right|_{\bar{\ell}_{0}} ^{\bar{\ell}_{f}}, \\
& a_{I \Omega^{\prime}}=a_{\Omega^{\prime} I}=-\left.\frac{1}{4} \sqrt{\frac{\bar{a}^{5}}{\mu^{3}}} \cos 2 \bar{\ell}\right|_{\bar{\ell}_{0}} ^{\bar{\ell}_{f}}, \\
& a_{\Omega^{\prime} \Omega^{\prime}}=\left.\sqrt{\frac{\overline{\bar{a}^{5}}}{\mu^{3}}}\left[\frac{1}{2} \bar{\ell}-\frac{1}{4} \sin 2 \bar{\ell}\right]\right|_{\bar{\ell}_{0}} ^{\bar{\ell}_{f}}, \\
& b_{\alpha}=\left.\varepsilon \sin ^{2} \bar{I} \cos 2 \bar{\ell}\right|_{\bar{\ell}_{0}} ^{\bar{\ell}_{f}},
\end{aligned}
$$




$$
\begin{aligned}
& b_{h}=\left.\varepsilon\left[\cos \bar{\ell}+\frac{1}{4} \sin ^{2} \bar{I}\left(-5 \cos \bar{\ell}+\frac{7}{3} \cos 3 \bar{\ell}\right)\right]\right|_{\bar{\ell}_{0}} ^{\bar{\ell}_{f}} \\
& b_{k}=\left.\varepsilon\left[\sin \bar{\ell}+\frac{1}{4} \sin ^{2} \bar{I}\left(-7 \sin \bar{\ell}+\frac{7}{3} \sin 3 \bar{\ell}\right)\right]\right|_{\bar{\ell}_{0}{ }^{\prime}} ^{\bar{\ell}_{f}} \\
& b_{I}=\left.\frac{\varepsilon}{4} \sin 2 \bar{I} \cos 2 \bar{\ell}\right|_{\bar{\ell}_{0}}{ }^{\bar{\ell}_{f}} \\
& b_{\Omega^{\prime}}=-\left.\frac{\varepsilon}{2} \sin 2 \bar{I}\left(\bar{\ell}-\frac{1}{2} \sin 2 \bar{\ell}\right)\right|_{\bar{\ell}_{0}{ }^{\prime}} ^{\bar{\ell}_{f}}
\end{aligned}
$$

with $\bar{\ell}_{f}=\bar{\ell}_{0}+\bar{n}\left(t_{f}-t_{0}\right), t_{0}$ is the initial time, $t_{f}$ is the final time, and $\varepsilon=(3 / 2) J_{2}\left(a_{e} / \bar{a}\right)^{2}$. The overbar denotes the orbital elements of the reference orbit.

The analytical solution defined by (5.4)-(5.7) is in agreement with the ones obtained through different approaches in $[6,10]$. In [6] the optimization problem is formulated with Gauss equations in nonsingular orbital elements as state equations, and in [10] Hori method is applied after the transformation of variables. Equations (5.4)-(5.7) represent a complete first-order analytical solution for optimal low-thrust limited-power transfers between close quasicircular nonequatorial orbits in a gravity field that includes the second zonal harmonic $J_{2}$ in the gravitational potential. These equations contain arbitrary constants of integration that must be determined to satisfy the two-point boundary value problem of going from the initial orbit at the time $t_{0}=0$ to the final orbit at the specified final time $t_{f}=T$. Since they are linear in these constants, the boundary value problem can be solved by simple techniques.

An approximate expression for the optimal thrust acceleration $\gamma^{*}$ can be obtained from (3.5) and (5.2), by using the expansions of the elliptic motion, and it is given, up to the zeroth order in eccentricity, by

$$
\begin{gathered}
r^{*}=\frac{1}{n a}\left\{\left(p_{h} \sin \ell-p_{k} \cos \ell\right) \mathbf{e}_{r}+2\left(p_{\alpha}+p_{h} \cos \ell+p_{k} \sin \ell\right) \mathbf{e}_{s}\right. \\
\left.+\left(p_{I} \cos \ell+p_{\Omega^{\prime}} \sin \ell\right) \mathbf{e}_{w}\right\} .
\end{gathered}
$$

According to Section 2, the optimal fuel consumption variable $J$ is determined by simple quadrature of the last differential equation in (2.3), with $r^{*}$ given by (5.8). An approximate expression for $J$ is given by

$$
\begin{gathered}
\Delta J=\frac{1}{2}\left\{a_{\alpha \alpha} p_{\alpha}^{2}+2 a_{\alpha h} p_{\alpha} p_{h}+2 a_{\alpha k} p_{\alpha} p_{k}+a_{h h} p_{h}^{2}+2 a_{h k} p_{h} p_{k}+a_{k k} p_{k}^{2}\right. \\
\left.+a_{I I} p_{I}^{2}+2 a_{h} \Omega^{\prime} p_{I} p_{\Omega^{\prime}}+a_{\Omega^{\prime} \Omega^{\prime}} p_{\Omega^{\prime}}^{2}\right\} .
\end{gathered}
$$

For transfers involving a large number of revolutions, (5.4)-(5.7) can be greatly simplified by neglecting the short periodic terms in comparison to the secular ones, and, 
the system of algebraic can be solved analytically. Thus,

$$
\begin{aligned}
p_{\alpha} & =\frac{1}{4} \sqrt{\frac{\mu^{3}}{\bar{a}^{5}}}\left(\frac{\Delta \alpha}{\Delta \bar{\ell}}\right), \\
p_{h} & =\frac{2}{5} \sqrt{\frac{\mu^{3}}{\bar{a}^{5}}}\left(\frac{\Delta h}{\Delta \bar{\ell}}\right), \\
p_{k} & =\frac{2}{5} \sqrt{\frac{\mu^{3}}{\bar{a}^{5}}}\left(\frac{\Delta k}{\Delta \bar{\ell}}\right), \\
p_{I} & =2 \sqrt{\frac{\mu^{3}}{\bar{a}^{5}}}\left(\frac{\Delta I}{\Delta \bar{\ell}}\right), \\
p_{\Omega^{\prime}}=2 \sqrt{\frac{\mu^{3}}{\bar{a}^{5}}} & {\left[\left(\frac{\Delta \Omega \sin \bar{I}}{\Delta \bar{\ell}}\right)+\frac{\varepsilon}{2} \sin 2 \bar{I}\right] . }
\end{aligned}
$$

Introducing these equations into (5.8) and (5.9), $\boldsymbol{r}^{*}$ and $J$ are obtained explicitly in terms of the imposed variations of the orbital elements, the perturbative effects due to the oblateness of the central body and the duration of the maneuver:

$$
\begin{aligned}
r^{*}=\frac{\mu}{\bar{a}^{2}}\{ & \frac{2}{5}\left(\frac{\Delta h}{\Delta \bar{\ell}} \sin \bar{\ell}-\frac{\Delta k}{\Delta \bar{\ell}} \cos \bar{\ell}\right) \mathbf{e}_{r}+\left(\frac{1}{2} \frac{\Delta \alpha}{\Delta \bar{\ell}}+\frac{4}{5}\left(\frac{\Delta h}{\Delta \bar{\ell}} \cos \bar{\ell}+\frac{\Delta k}{\Delta \bar{\ell}} \sin \bar{\ell}\right)\right) \mathbf{e}_{s} \\
& \left.+2\left(\frac{\Delta I}{\Delta \bar{\ell}} \cos \bar{\ell}+\left[\frac{\Delta \Omega}{\Delta \bar{\ell}}+\varepsilon \cos \bar{I}\right] \sin \bar{I} \sin \bar{\ell}\right) \mathbf{e}_{w}\right\} \\
\frac{\Delta J}{\Delta \bar{\ell}}= & \frac{1}{4} \sqrt{\frac{\mu^{3}}{\bar{a}^{5}}}\left\{\frac{1}{2}\left(\frac{\Delta \alpha}{\Delta \bar{\ell}}\right)^{2}+\frac{4}{5}\left(\frac{\Delta h^{2}+\Delta k^{2}}{\Delta \bar{\ell}^{2}}\right)+4\left(\left(\frac{\Delta I}{\Delta \bar{\ell}}\right)^{2}+\left[\frac{\Delta \Omega}{\Delta \bar{\ell}}+\varepsilon \cos \bar{I}\right]^{2} \sin ^{2} \bar{I}\right)\right\}
\end{aligned}
$$

\subsection{Transfers between Close Quasicircular Near-Equatorial Orbits}

Consider the Mathieu transformation [33] defined by

$$
\begin{gathered}
a^{\prime \prime}=a^{\prime}, \quad \xi^{\prime \prime}=e^{\prime} \cos \left(\omega^{\prime}+\Omega^{\prime}\right), \quad \eta^{\prime \prime}=e^{\prime} \sin \left(\omega^{\prime}+\Omega^{\prime}\right), \\
P^{\prime \prime}=\sin \left(\frac{I^{\prime}}{2}\right) \cos \Omega^{\prime}, \quad Q^{\prime \prime}=\sin \left(\frac{I^{\prime}}{2}\right) \sin \Omega^{\prime}, \quad \lambda^{\prime \prime}=M^{\prime}+\omega^{\prime}+\Omega^{\prime},
\end{gathered}
$$




$$
\begin{gathered}
p_{a}^{\prime \prime}=p_{a}, \\
p_{\xi}^{\prime \prime}=p_{e}^{\prime} \cos \left(\omega^{\prime}+\Omega^{\prime}\right)-\left(\frac{p_{\omega}^{\prime}-p_{M}^{\prime}}{e^{\prime}}\right) \sin \left(\omega^{\prime}+\Omega^{\prime}\right), \\
p_{\eta}^{\prime \prime}=p_{e}^{\prime} \sin \left(\omega^{\prime}+\Omega^{\prime}\right)+\left(\frac{p_{\omega}^{\prime}-p_{M}^{\prime}}{e^{\prime}}\right) \cos \left(\omega^{\prime}+\Omega^{\prime}\right), \\
p_{P}^{\prime \prime}=p_{I}^{\prime} \frac{2 \cos \Omega^{\prime}}{\cos \left(I^{\prime} / 2\right)}-\left(p_{\Omega}^{\prime}-p_{\omega}^{\prime}\right) \frac{\sin \Omega^{\prime}}{\sin \left(I^{\prime} / 2\right)}, \\
p_{Q}^{\prime \prime}=p_{I}^{\prime} \frac{2 \sin \Omega^{\prime}}{\cos \left(I^{\prime} / 2\right)}+\left(p_{\Omega}^{\prime}-p_{\omega}^{\prime}\right) \frac{\cos \Omega^{\prime}}{\sin \left(I^{\prime} / 2\right)}, \\
p_{\lambda}^{\prime \prime}=p_{M}^{\prime} .
\end{gathered}
$$

The new set of canonical variables, designated by the double prime, is nonsingular for circular equatorial orbits.

Introducing (5.14) into (4.6) and using the expansions of the elliptic motion in terms of eccentricity and mean anomaly [31], one finds that the new averaged maximum Hamiltonian and the generating function are written up to the zeroth order in eccentricity and first-order in inclination as

$$
\begin{aligned}
F_{1}= & \frac{3}{2} n J_{2}\left(\frac{a_{e}}{a}\right)^{2}\left(Q p_{P}-P p_{Q}\right)+\frac{a}{2 \mu}\left\{4 a^{2} p_{a}^{2}+\frac{5}{2}\left(p_{\xi}^{2}+p_{\eta}^{2}\right)+\frac{1}{8}\left(p_{P}^{2}+p_{Q}^{2}\right)\right\}, \\
S_{1}= & \frac{3}{2} J_{2}\left(\frac{a_{e}}{a}\right)^{2}\left\{p_{\xi} \cos \lambda+p_{\eta} \sin \lambda+\frac{1}{2}\left(Q p_{P}+P p_{Q}\right) \sin 2 \lambda+\frac{1}{2}\left(P p_{P}-Q p_{Q}\right) \cos 2 \lambda\right\} \\
& +\frac{1}{2} \sqrt{\frac{a^{5}}{\mu^{3}}}\left\{8 a p_{a}\left(p_{\xi} \sin \lambda-p_{\eta} \cos \lambda\right)+\left[\frac{3}{4}\left(p_{\xi}^{2}-p_{\eta}^{2}\right)+\frac{1}{16}\left(p_{P}^{2}-p_{Q}^{2}\right)\right] \sin 2 \lambda\right. \\
& \left.-\left[\frac{3}{2} p_{\xi} p_{\eta}+\frac{1}{8} p_{P} p_{Q}\right] \cos 2 \lambda\right\} .
\end{aligned}
$$

Double prime is omitted to simplify the notation.

For transfers between close orbits, $F_{1}$ and $S_{1}$ can be linearized around a suitable reference orbit, and an approximate first-order analytical solution can be determined. This solution is given by

$$
\Delta x=C p_{0}+D,
$$

where $\Delta x=[\Delta \alpha \Delta \xi \Delta \eta \Delta P \Delta Q]^{T}, p_{0}$ is the $5 \times 1$ vector of initial value of the adjoint variables, $C$ is a $5 \times 5$ matrix concerning the optimal thrust acceleration, and $D$ is a $5 \times 1$ vector containing the perturbative effects of the oblateness of the central body. The matrix $C$ and the vector $D$ 
are given by

$$
C=\left[\begin{array}{ccccc}
c_{\alpha \alpha} & c_{\alpha \xi} & c_{\alpha \eta} & 0 & 0 \\
c_{\xi \alpha} & c_{\xi \xi} & c_{\xi \eta} & 0 & 0 \\
c_{\eta \alpha} & c_{\eta \xi} & c_{\eta \eta} & 0 & 0 \\
0 & 0 & 0 & c_{P P} & c_{P Q} \\
0 & 0 & 0 & c_{Q P} & c_{Q Q}
\end{array}\right],
$$

$$
D=\left[\begin{array}{c}
0 \\
d_{\xi} \\
d_{\eta} \\
d_{P} \\
d_{Q}
\end{array}\right]
$$

where

$$
\begin{aligned}
& c_{\alpha \alpha}=\left.4 \sqrt{\frac{\overline{a^{5}}}{\mu^{3}}} \bar{\lambda}\right|_{\bar{\lambda}_{0}} ^{\bar{\lambda}_{f}}, \quad c_{\alpha \xi}=c_{\xi \alpha}=\left.4 \sqrt{\frac{\bar{a}^{5}}{\mu^{3}}} \sin \bar{\lambda}\right|_{\bar{\lambda}_{0}} ^{\bar{\lambda}_{f}}, \\
& c_{\alpha \eta}=c_{\eta \alpha}=-\left.4 \sqrt{\frac{\bar{a}^{5}}{\mu^{3}}} \cos \bar{\lambda}\right|_{\bar{\lambda}_{0}} ^{\bar{\lambda}_{f}}, \quad c_{\xi \xi}=\left.\sqrt{\frac{\bar{a}^{5}}{\mu^{3}}}\left[\frac{5}{2} \bar{\lambda}+\frac{3}{4} \sin 2 \bar{\lambda}\right]\right|_{\bar{\lambda}_{0}} ^{\bar{\lambda}_{f}}, \\
& c_{\xi \eta}=c_{\eta \xi}=-\left.\frac{3}{4} \sqrt{\frac{\bar{a}^{5}}{\mu^{3}}} \cos 2 \bar{\lambda}\right|_{\bar{\lambda}_{0}} ^{\bar{\lambda}_{f}}, \quad c_{\eta \eta}=\left.\sqrt{\frac{\bar{a}^{5}}{\mu^{3}}}\left[\frac{5}{2} \bar{\lambda}-\frac{3}{4} \sin 2 \bar{\lambda}\right]\right|_{\bar{\lambda}_{0}} ^{\bar{\lambda}_{f}}, \\
& c_{P P}=\frac{1}{8} \sqrt{\frac{\bar{a}^{5}}{\mu^{3}}}\left[\Delta \overline{\mathcal{\lambda}} \cos \varepsilon \Delta \overline{\mathcal{\lambda}}+\frac{1}{2} \sin \left((2+\varepsilon) \bar{\lambda}_{f}-\varepsilon \bar{\lambda}_{0}\right)-\frac{1}{2} \sin \left((2+\varepsilon) \bar{\lambda}_{0}-\varepsilon \bar{\lambda}_{f}\right)\right], \\
& c_{P Q}=\frac{1}{8} \sqrt{\frac{\bar{a}^{5}}{\mu^{3}}}\left[\Delta \bar{\lambda} \sin \varepsilon \Delta \bar{\lambda}-\frac{1}{2} \cos \left((2+\varepsilon) \bar{\lambda}_{f}-\varepsilon \bar{\lambda}_{0}\right)+\frac{1}{2} \cos \left((2+\varepsilon) \bar{\lambda}_{0}-\varepsilon \bar{\lambda}_{f}\right)\right], \\
& c_{Q P}=\frac{1}{8} \sqrt{\frac{\bar{a}^{5}}{\mu^{3}}}\left[-\Delta \bar{\lambda} \sin \varepsilon \Delta \bar{\lambda}-\frac{1}{2} \cos \left((2+\varepsilon) \bar{\lambda}_{f}-\varepsilon \bar{\lambda}_{0}\right)+\frac{1}{2} \cos \left((2+\varepsilon) \bar{\lambda}_{0}-\varepsilon \bar{\lambda}_{f}\right)\right],
\end{aligned}
$$




$$
\begin{aligned}
c_{Q Q}= & \frac{1}{8} \sqrt{\frac{\bar{a}^{5}}{\mu^{3}}}\left[\Delta \bar{\lambda} \cos \varepsilon \Delta \bar{\lambda}-\frac{1}{2} \sin \left((2+\varepsilon) \bar{\lambda}_{f}-\varepsilon \bar{\lambda}_{0}\right)+\frac{1}{2} \sin \left((2+\varepsilon) \bar{\lambda}_{0}-\varepsilon \bar{\lambda}_{f}\right)\right], \\
d_{\xi}= & \left.\frac{3}{2} J_{2}\left(\frac{a_{e}}{\bar{a}}\right)^{2} \cos \bar{\lambda}\right|_{\bar{\lambda}_{0}} ^{\bar{\lambda}_{f}}, \quad d_{\eta}=\left.\frac{3}{2} J_{2}\left(\frac{a_{e}}{\bar{a}}\right)^{2} \sin \bar{\lambda}\right|_{\bar{\lambda}_{0}} ^{\bar{\lambda}_{f}}, \\
d_{P}= & P_{0}\left\{\left[\cos \varepsilon \Delta \bar{\lambda}-\frac{1}{2} \varepsilon \cos \left(\varepsilon \bar{\lambda}_{f}-(2+\varepsilon) \bar{\lambda}_{0}\right)+\frac{1}{2} \varepsilon \cos \left(\varepsilon \bar{\lambda}_{0}-(2+\varepsilon) \bar{\lambda}_{f}\right)\right]-1\right\} \\
& +Q_{0}\left[\sin \varepsilon \Delta \bar{\lambda}+\frac{1}{2} \varepsilon \sin \left(\varepsilon \bar{\lambda}_{f}-(2+\varepsilon) \bar{\lambda}_{0}\right)-\frac{1}{2} \varepsilon \sin \left(\varepsilon \bar{\lambda}_{0}-(2+\varepsilon) \bar{\lambda}_{f}\right)\right], \\
d_{Q}= & Q_{0}\left\{\left[\cos \varepsilon \Delta \bar{\lambda}+\frac{1}{2} \varepsilon \cos \left(\varepsilon \bar{\lambda}_{f}-(2+\varepsilon) \bar{\lambda}_{0}\right)-\frac{1}{2} \varepsilon \cos \left(\varepsilon \bar{\lambda}_{0}-(2+\varepsilon) \bar{\lambda}_{f}\right)\right]-1\right\} \\
& +P_{0}\left[-\sin \varepsilon \Delta \bar{\lambda}+\frac{1}{2} \varepsilon \sin \left(\varepsilon \bar{\lambda}_{f}-(2+\varepsilon) \bar{\lambda}_{0}\right)-\frac{1}{2} \varepsilon \sin \left(\varepsilon \bar{\lambda}_{0}-(2+\varepsilon) \bar{\lambda}_{f}\right)\right] .
\end{aligned}
$$

The adjoint variables are

$$
\begin{gathered}
p_{a}=p_{a_{0}}, \\
p_{\xi}=p_{\xi_{0}}, \\
p_{\eta}=p_{\eta_{0}}, \\
p_{P}=p_{P_{0}} \cos \varepsilon \Delta \overline{\mathcal{\lambda}}+p_{Q_{0}} \sin \varepsilon \Delta \bar{\lambda} \\
p_{Q}=-p_{P_{0}} \sin \varepsilon \Delta \bar{\lambda}+p_{Q_{0}} \cos \varepsilon \Delta \bar{\lambda}
\end{gathered}
$$

where $\Delta \bar{\lambda}=\bar{\lambda}_{f}-\bar{\lambda}_{0}=\bar{n}\left(t_{f}-t_{0}\right)$. The overbar denotes the orbital elements of the reference orbit. Equations (5.20) do not contain higher order terms in $\varepsilon$ as appear implicitly in the solution presented in [7].

The analytical solution defined by (5.17)-(5.21) is in agreement with the ones obtained through different approaches in [7, 10]. In [7] the optimization problem is formulated with Gauss equations in nonsingular orbital elements as state equations, and in [10] Hori method is applied after the transformation of variables. Equations (5.17)-(5.21) represent a complete first-order analytical solution for optimal low-thrust limited-power transfers between close quasicircular near-equatorial orbits in a gravity field that includes the second zonal harmonic $J_{2}$ in the gravitational potential. These equations contain arbitrary constants of integration that must be determined to satisfy the two-point boundary value problem of going from the initial orbit at the time $t_{0}=0$ to the final orbit at the specified final time $t_{f}=T$. Since they are linear in these constants, the boundary value problem can be solved by simple techniques. 
As described in Section 5.1, approximate expressions can be obtained for the optimal thrust acceleration $r^{*}$ and the fuel consumption $J$. These expressions are given by

$$
\begin{aligned}
& r^{*}=\frac{1}{n a}\left\{\left(p_{\xi} \sin \lambda-p_{\eta} \cos \lambda\right) \mathbf{e}_{r}+2\left(p_{\alpha}+p_{\xi} \cos \lambda+p_{\eta} \sin \lambda\right) \mathbf{e}_{s}+\frac{1}{2}\left(p_{P} \cos \lambda+p_{Q} \sin \lambda\right) \mathbf{e}_{w}\right\}, \\
& \Delta J=\frac{1}{2}\left\{c_{\alpha \alpha} p_{\alpha}^{2}+2 c_{\alpha \xi} p_{\alpha} p_{\xi}+2 c_{\alpha \eta} p_{\alpha} p_{\eta}+c_{\xi \xi} p_{\xi}^{2}+2 c_{\xi \eta} p_{\xi} p_{\eta}+c_{\eta \eta} p_{\eta}^{2}\right. \\
& \left.+c_{P P} p_{P}^{2}+\left(c_{P Q}+c_{Q P}\right) p_{P} p_{Q}+c_{Q Q} p_{Q}^{2}\right\} .
\end{aligned}
$$

For transfers involving a large number of revolutions, (5.17)-(5.21) can be greatly simplified by neglecting the short periodic terms in comparison to the secular ones, and, the system of algebraic can be solved analytically. Thus,

$$
\begin{aligned}
& p_{\alpha}=\frac{1}{4} \sqrt{\frac{\mu^{3}}{\bar{a}^{5}}}\left(\frac{\Delta \alpha}{\Delta \bar{\jmath}}\right) \\
& p_{\xi}=\frac{2}{5} \sqrt{\frac{\mu^{3}}{\bar{a}^{5}}}\left(\frac{\Delta \xi}{\Delta \bar{l}}\right) \\
& p_{\eta}=\frac{2}{5} \sqrt{\frac{\mu^{3}}{\bar{a}^{5}}}\left(\frac{\Delta \eta}{\Delta \bar{l}}\right) \\
& p_{P}=\sqrt{\frac{\mu^{3}}{\bar{a}^{5}}} \frac{8}{\Delta \bar{l}}\left[\left(P_{0}+\Delta P\right) \cos (\varepsilon \Delta \bar{l})-\left(Q_{0}+\Delta Q\right) \sin (\varepsilon \Delta \bar{l})-P_{0}\right] \\
& p_{Q}=\sqrt{\frac{\mu^{3}}{\bar{a}^{5}}} \frac{8}{\Delta \bar{\lambda}}\left[\left(Q_{0}+\Delta Q\right) \cos (\varepsilon \Delta \bar{\lambda})+\left(P_{0}+\Delta P\right) \sin (\varepsilon \Delta \bar{l})-Q_{0}\right]
\end{aligned}
$$

The subscript denoting the constants is omitted. Introducing these equations into (5.22), $\boldsymbol{r}^{*}$ and $J$ are obtained explicitly in terms of the imposed variations of the orbital elements, the perturbative effects due to the oblateness of the central body and the duration of the maneuver:

$$
\begin{aligned}
r^{*}=\frac{\mu}{\bar{a}^{2}}\{ & \frac{2}{5}\left(\frac{\Delta \xi}{\Delta \bar{\jmath}} \sin \bar{\lambda}-\frac{\Delta \eta}{\Delta \bar{\jmath}} \cos \bar{\lambda}\right) \mathbf{e}_{r}+\left(\frac{1}{2} \frac{\Delta \alpha}{\Delta \bar{\lambda}}+\frac{4}{5}\left(\frac{\Delta \xi}{\Delta \bar{\lambda}} \cos \bar{\lambda}+\frac{\Delta \eta}{\Delta \bar{\lambda}} \sin \bar{\lambda}\right)\right) \mathbf{e}_{s} \\
& \left.+\frac{4}{\Delta \bar{\lambda}}\left[P_{f} \cos \left(\bar{\lambda}+\theta_{f}\right)-P_{0} \cos \left(\bar{\lambda}+\theta_{0}\right)+Q_{f} \sin \left(\bar{\lambda}+\theta_{f}\right)-Q_{0} \sin \left(\bar{\lambda}+\theta_{0}\right)\right] \mathbf{e}_{w}\right\},
\end{aligned}
$$




$$
\begin{aligned}
& \frac{\Delta J}{\Delta \bar{\jmath}}=\frac{1}{4} \sqrt{\frac{\mu^{3}}{\bar{a}^{5}}}\left\{\frac{1}{2}\left(\frac{\Delta \alpha}{\Delta \bar{l}}\right)^{2}+\frac{4}{5}\left(\frac{\Delta \xi^{2}+\Delta \eta^{2}}{\Delta \bar{\jmath}^{2}}\right)\right. \\
& +16 \cos (\varepsilon \Delta \bar{\lambda})\left\{\left(\frac{\Delta P^{2}+\Delta Q^{2}}{\Delta \bar{\jmath}^{2}}\right)+\frac{4}{\Delta \bar{\jmath}^{2}} \sin \left(\frac{\varepsilon \Delta \bar{\jmath}}{2}\right)\right. \\
& \times\left[P_{f}\left(P_{0} \sin \left(\frac{\varepsilon \Delta \bar{\lambda}}{2}\right)-Q_{0} \cos \left(\frac{\varepsilon \Delta \bar{\jmath}}{2}\right)\right)\right. \\
& \left.\left.\left.+Q_{f}\left(P_{0} \cos \left(\frac{\varepsilon \Delta \bar{\lambda}}{2}\right)+Q_{0} \sin \left(\frac{\varepsilon \Delta \bar{\lambda}}{2}\right)\right)\right]\right\}\right\},
\end{aligned}
$$

with $\theta_{0}=\varepsilon \bar{n}\left(t-t_{0}\right)$ and $\theta_{f}=\varepsilon \bar{n}\left(t-t_{f}\right)$.

\subsection{General Remarks on Optimal Transfers between Close Quasicircular Orbits}

Considering the analytical solutions described above, general remarks about the effects of the oblateness of the central body on optimal transfers between close quasicircular orbits can be stated.

(1) Matrices $A$ and $C$, defined by (5.5) and (5.18), respectively, can be decomposed into two square matrices $3 \times 3$ and $2 \times 2$. This fact shows that there exists an uncoupling between the in-plane modifications and the rotation of the orbital plane. Similar results were obtained by Edelbaum [1] and Marec [3] for transfers in a Newtonian central field.

(2) There is a normal component of the optimal thrust acceleration that counteracts the perturbative effects due to the oblateness of the central body. This normal component is proportional to $\varepsilon$.

(3) In general, the maneuvers in a noncentral gravity field are more expensive than the maneuvers in Newtonian central field, taking into account that the perturbative effects due to the oblateness of the central body must be counteracted.

(4) Fuel can be saved for long-time maneuvers which involves modification of the longitude of the ascending node if the terminal orbits are direct $\left(0^{\circ}<\bar{I}<90^{\circ}\right)$ and $\Delta \Omega<0$, and if the terminal orbits are retrograde $\left(90^{\circ}<\bar{I}<180^{\circ}\right)$ and $\Delta \Omega>0$.

(5) The extra consumption needed to counteract the perturbative effects due to the oblateness of the central body reaches its maximum for maneuvers between orbits with $45^{\circ}$ or $135^{\circ}$ of inclination and its minimum (null extra consumption) for maneuvers between equatorial or polar orbits (the gravity field is symmetric in these cases).

(6) As the transfer time increases, the extra fuel consumption increases.

(7) The extra fuel consumption is greater for transfers between low orbits. 
Table 1: Maneuver of modification of all orbital elements.

\begin{tabular}{lccccc}
\hline \multirow{2}{*}{$T=t_{f}-t_{0}$} & \multicolumn{2}{c}{$a_{e} / a_{0}$} & \multicolumn{2}{c}{ Maneuver of modification of all orbital elements } \\
& \multicolumn{2}{c}{ Initial orbit } & Final orbit & Orbital elements \\
\hline \multirow{2}{*}{100} & 0.950 & 0.900 & & & \\
& 0.850 & 0.800 & & & \\
& 0.750 & 0.700 & 1.000 & 0.150 & $e$ \\
200 & 0.650 & 0.600 & 0.0025 & 0.050 & $\Omega$ \\
& 0.550 & 0.500 & $30.00^{\circ}$ & $35.0^{\circ}$ & $\omega$ \\
250 & 0.450 & 0.400 & $30.00^{\circ}$ & $37.5^{\circ}$ & \\
& 0.350 & 0.300 & $60.00^{\circ}$ & $62.50^{\circ}$ & \\
\hline
\end{tabular}

\section{Optimal Long-Time Transfers between Arbitrary Orbits}

In this section the two-point boundary-value problem for long-time transfers between arbitrary orbits is formulated. In Section 7, this boundary-problem problem will be solved numerically through a shooting method, and the solution will be compared to the analytical ones in the case of orbits with small eccentricities.

Since the averaged maximum Hamiltonian, given by (4.6), becomes singular for circular and/or equatorial orbits, a set of nonsingular elements is introduced.

Consider the Mathieu transformation [33] defined by

$$
\begin{gathered}
a^{\prime \prime}=a^{\prime}, \\
\xi^{\prime \prime}=e^{\prime} \cos \left(\omega^{\prime}+\Omega^{\prime}\right), \\
\eta^{\prime \prime}=e^{\prime} \sin \left(\omega^{\prime}+\Omega^{\prime}\right), \\
P^{\prime \prime}=\sin \left(\frac{I^{\prime}}{2}\right) \cos \Omega^{\prime}, \\
Q^{\prime \prime}=\sin \left(\frac{I^{\prime}}{2}\right) \sin \Omega^{\prime}, \\
p_{a}^{\prime \prime}=p_{a}^{\prime}, \\
p_{\xi}^{\prime \prime}=p_{e}^{\prime} \cos \left(\omega^{\prime}+\Omega^{\prime}\right)-\left(\frac{p_{\omega}^{\prime}-p_{M}^{\prime}}{e^{\prime}}\right) \sin \left(\omega^{\prime}+\Omega^{\prime}\right), \\
p_{\eta}^{\prime \prime}=p_{e}^{\prime} \sin \left(\omega^{\prime}+\Omega^{\prime}\right)+\left(\frac{p_{\omega}^{\prime}-p_{M}^{\prime}}{e^{\prime}}\right) \cos \left(\omega^{\prime}+\Omega^{\prime}\right), \\
p_{P}^{\prime \prime}=p_{I}^{\prime} \frac{2 \cos \Omega^{\prime}}{\cos \left(I^{\prime} / 2\right)}-\left(p_{\Omega}^{\prime}-p_{\omega}^{\prime}\right) \frac{\sin \Omega^{\prime}}{\sin \left(I^{\prime} / 2\right)}, \\
p_{Q}^{\prime \prime}=p_{I}^{\prime} \frac{2 \sin \Omega^{\prime}}{\cos \left(I^{\prime} / 2\right)}+\left(p_{\Omega}^{\prime}-p_{\omega}^{\prime}\right) \frac{\cos \Omega^{\prime}}{\sin \left(I^{\prime} / 2\right)} .
\end{gathered}
$$

This transformation of variables is the same one given by (5.12), excepting the fast-phase, which is unnecessary (short-periodic terms have been eliminated). Double prime designates the new variables. 
Introducing (6.2) into (4.6), $F_{1}$ is written as

$$
\begin{aligned}
F_{1}=-\frac{3}{2} n J_{2}\left(\frac{a_{e}}{a}\right)^{2}\left(1-\xi^{2}-\eta^{2}\right)^{-2}\{ & \left(1-2\left(P^{2}+Q^{2}\right)\right)\left[\left(\xi p_{\eta}-\eta p_{\xi}\right)+\left(P p_{Q}-Q p_{P}\right)\right] \\
& \left.+\frac{1}{2}\left(1-5\left(1-2\left(P^{2}+Q^{2}\right)\right)^{2}\right)\left(\xi p_{\eta}-\eta p_{\xi}\right)\right\} \\
+\frac{a}{2 \mu}\left\{4 a^{2} p_{a}^{2}+p_{\xi}^{2}\left(\frac{5}{2}-\frac{5}{2} \xi^{2}-2 \eta^{2}\right)+p_{\eta}^{2}\left(\frac{5}{2}-\frac{5}{2} \eta^{2}-2 \xi^{2}\right)-\xi \eta p_{\xi} p_{\eta}\right. & \\
+\frac{1}{2}\left(1-\xi^{2}-\eta^{2}\right)^{-1}\left\{\left(1+\frac{3}{2} \xi^{2}+\frac{3}{2} \eta^{2}\right)\right. & \\
\times & {\left[\frac{1}{4}\left(p_{P}^{2}+p_{Q}^{2}\right)-\frac{1}{4}\left(P p_{P}+Q p_{Q}\right)^{2}+\frac{1}{4}\left(1-P^{2}-Q^{2}\right)^{-1}\right.} \\
\times & {\left[\left(Q p_{P}-P p_{Q}\right)^{2}+4\left(\xi p_{\eta}-\eta p_{\xi}\right)\left(P p_{Q}-Q p_{P}\right)\right.} \\
\left.\left.+4\left(P^{2}+Q^{2}\right)\left(\xi p_{\eta}-\eta p_{\xi}\right)^{2}\right]\right] & \\
& -\frac{5}{8}\left(1-P^{2}-Q^{2}\right)^{-1}\left[\left(\xi^{2}-\eta^{2}\right)\left(P^{2}-Q^{2}\right)+4 \xi \eta P Q\right] \\
\times & {\left[\left[2\left(\xi p_{\eta}-\eta p_{\xi}\right)+\left(P p_{Q}-Q p_{P}\right)\right]^{2}+\left(1-P^{2}-Q^{2}\right)\left(p_{P}^{2}+p_{Q}^{2}\right)\right] } \\
- & \frac{5}{2} \xi \eta p_{P} p_{Q}+\frac{5}{8}\left(\xi^{2}-\eta^{2}\right)\left(p_{P}^{2}-p_{Q}^{2}\right) \\
+ & \left.\frac{5}{2}\left(\xi p_{\eta}-\eta p_{\xi}\right)\left[-\left(\xi^{2}-\eta^{2}\right)\left(P p_{Q}+Q p_{P}\right)+2 \xi \eta\left(P p_{P}-Q p_{Q}\right)\right]\right\} .
\end{aligned}
$$

Double prime is omitted to simplify the notation. Note that (5.15) is a simplification of (6.3) for quasicircular near-equatorial orbits.

The two-point boundary-value problem for long-time transfers between arbitrary orbits is formulated by the following system of canonical differential equations:

$$
\frac{d x}{d t}=\left(\frac{\partial F_{1}}{\partial p}\right)^{T}, \quad \frac{d p}{d t}=-\left(\frac{\partial F_{1}}{\partial x}\right)^{T}
$$

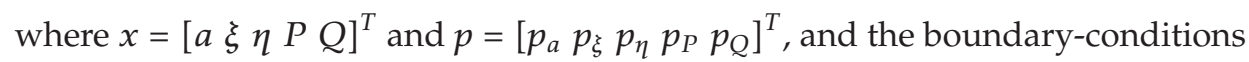

$$
\begin{aligned}
& a\left(t_{0}\right)=a_{0}, \quad \xi\left(t_{0}\right)=\xi_{0}, \quad \eta\left(t_{0}\right)=\eta_{0}, \quad P\left(t_{0}\right)=P_{0}, \quad Q\left(t_{0}\right)=Q_{0}, \\
& a\left(t_{f}\right)=a_{f}, \quad \xi\left(t_{f}\right)=\xi_{f}, \quad \eta\left(t_{f}\right)=\eta_{f}, \quad P\left(t_{f}\right)=P_{f}, \quad Q\left(t_{f}\right)=Q_{f},
\end{aligned}
$$

with initial time $t_{0}=0$ and final time $t_{f}=T$. The explicit form of state and adjoint equations is derived by using MAPLE Software 9, and they are not presented in text because they are extensive. 
Table 2: Small and moderate amplitude maneuvers.

\begin{tabular}{ccccr}
\hline$a_{e} / a_{0}$ & $T=t_{f}-t_{0}$ & Orbital elements of initial orbit & \multicolumn{2}{c}{$\rho=y_{f} / y_{0}$} \\
\hline \multirow{4}{*}{0.975} & 100 & $a=1.0$ & 1.05 & 1.10 \\
& 150 & $e=0.0025$ & 1.15 & 1.20 \\
& 200 & $I=30.00^{\circ}$ & 1.25 & 1.30 \\
& 250 & $\Omega=30.00^{\circ}$ & 1.35 & 1.40 \\
& - & $\omega=60.00^{\circ}$ & 1.45 & 1.50 \\
\hline
\end{tabular}

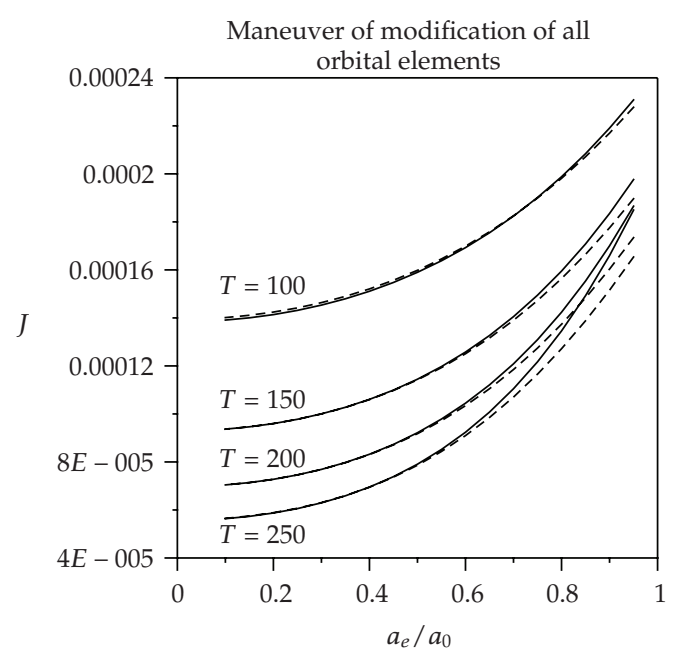

Figure 3: Consumption for maneuver of modification of all orbital elements.

\section{Results}

In this section, numerical and analytical results are compared for maneuvers described in Tables 1 and 2. Earth is the central body, such that $J_{2}=1.0826 \times 10^{-3}$. In Table 1, orbital elements of initial and final orbits are defined for a maneuver of modification of all orbital elements considering different values of transfer durations $T=t_{f}-t_{0}$ and ratios $a_{e} / a_{0}$. In Table 2, orbital elements of initial orbit, values of transfer duration and ratio $\rho=y_{f} / y_{0}$, where $y$ denotes the orbital element that is changed in the maneuver, are defined for small (orbit corrections) and moderate amplitude maneuvers of modification of semimajor axis and inclination of the orbital plane. Results are presented in Figures 3 through 8 using canonical units. In these units, $a_{0}=1.0$ and $\mu=1.0$. For example, in metric units, taking $a_{e}=6378.2 \mathrm{~km}, \rho=0.950$ corresponds to $a_{0}=6713.89 \mathrm{~km}$, and $T=100$ (time units) corresponds to 24.20 hours (1.008 day), and $\rho=0.150$ corresponds to $a_{0}=42521.34 \mathrm{~km}$, and $T=250$ (time units) corresponds to 964.45 hours (40.185 days). Thus, maneuvers described in the tables below have duration of 1 to 74 days, approximately. On the other hand, considering that the value of $\left|\gamma^{*}\right|$ is approximately given by $\sqrt{2 \Delta J / T}$ (short periodic terms are neglected), $\left|r^{*}\right|$ has values between $1.0 \times 10^{-4}$ and $4.0 \times 10^{-3}$ (canonical units) for all results presented in Figures 3-5. These values of magnitude of the thrust acceleration are typical for LP systems $[5,27]$. 


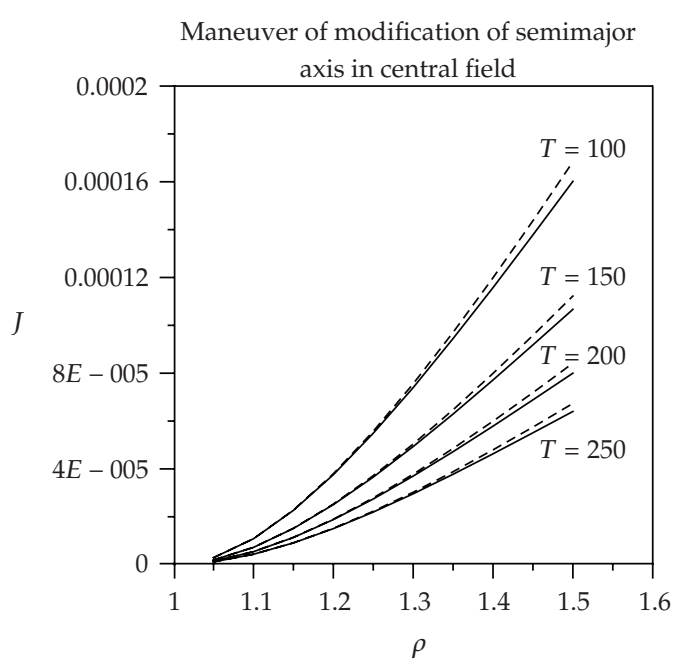

(a)

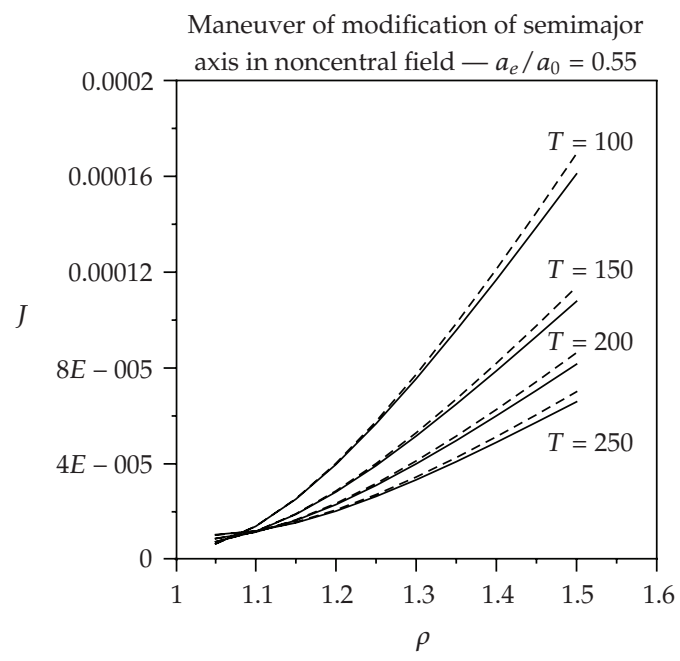

(c)

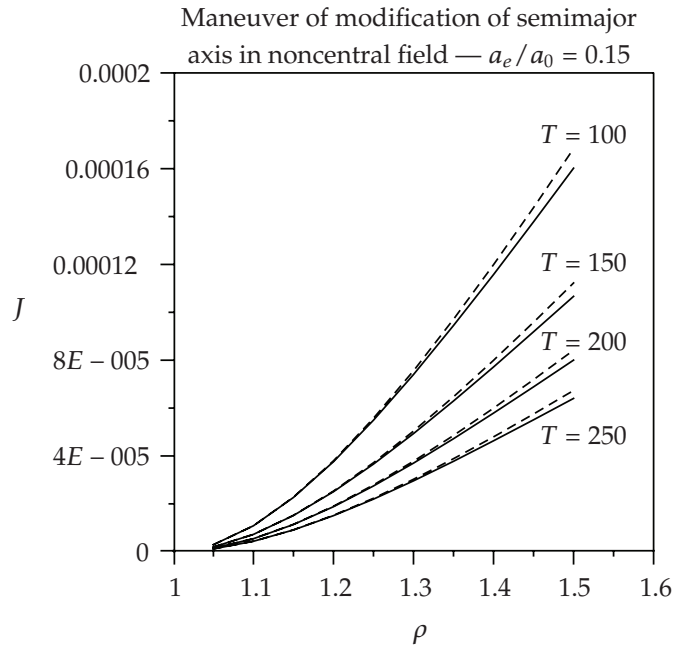

(b)

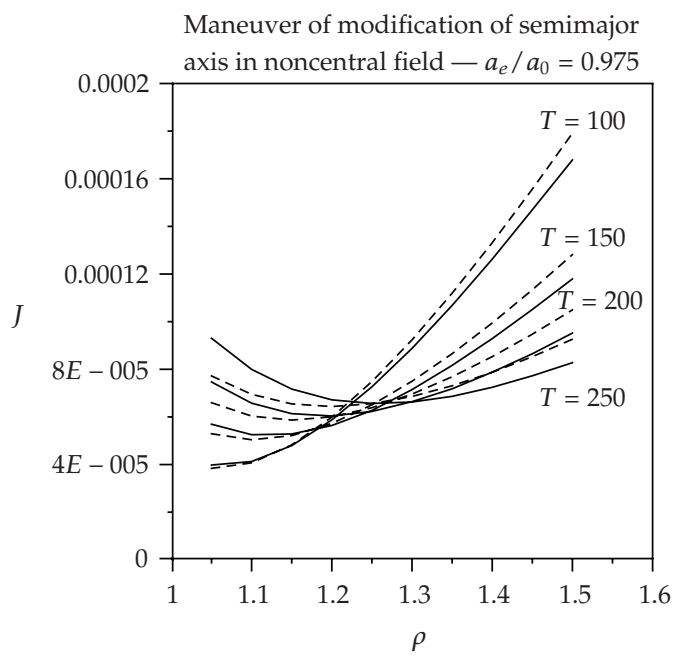

(d)

Figure 4: Consumption for maneuver of modification of semimajor axis.

Figure 3 shows the consumption $J$ as function of ratio $a_{e} / a_{0}$ and values of $T$ for the maneuver of modification of all orbital elements described in Table 1. Figures 4 and 5 show the consumption $J$ as function of ratio $\rho=y_{f} / y_{0}$ and $T$ for the small and moderate amplitude maneuvers described in Table 2, considering that the maneuvers are performed in central or noncentral gravity field. In these figures, solid line represents analytical solution, and dashed line represents numerical solution. Analytical solutions, including short periodic terms, are obtained by solving the linear system of algebraic equations given by (5.4)-(5.7) (transfer between nonequatorial orbits) with the semimajor axis and the inclination of the reference orbit defined by $\bar{a}=\left(a_{0}+a_{f}\right) / 2$ and $\bar{I}=\left(I_{0}+I_{f}\right) / 2$, respectively. In order to include the short periodic terms, it is assumed that the initial position of the vehicle is the pericenter of 


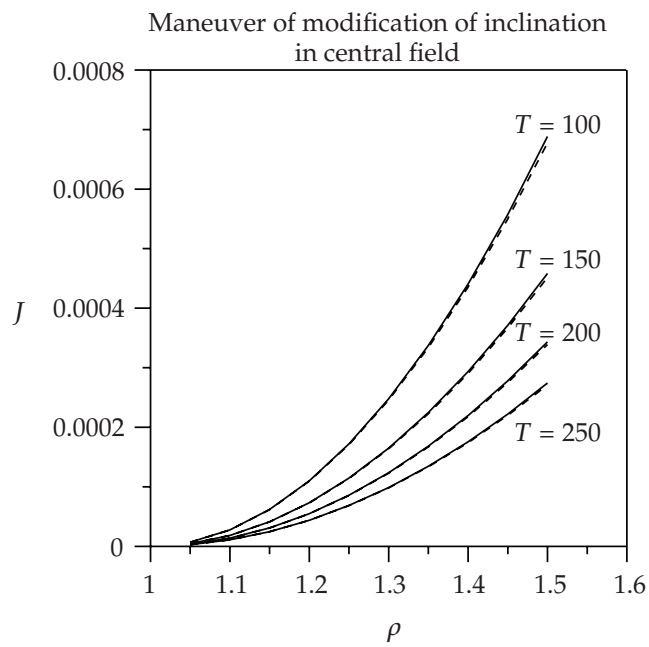

(a)

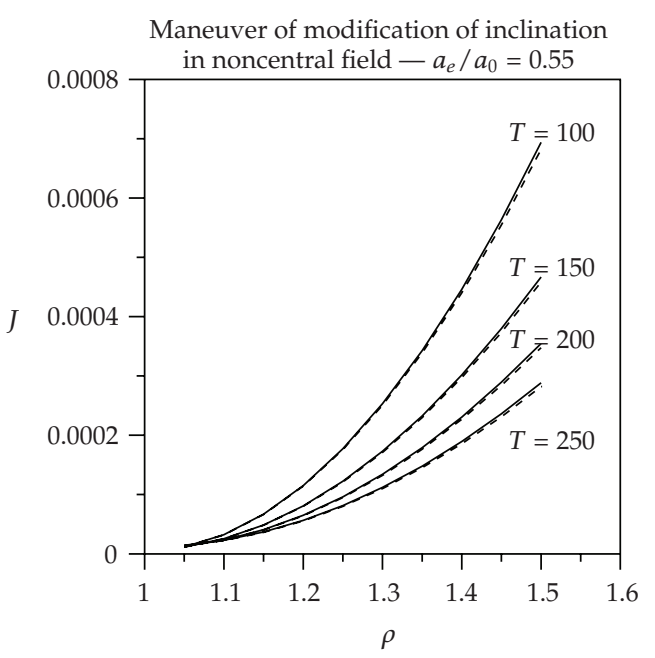

(c)

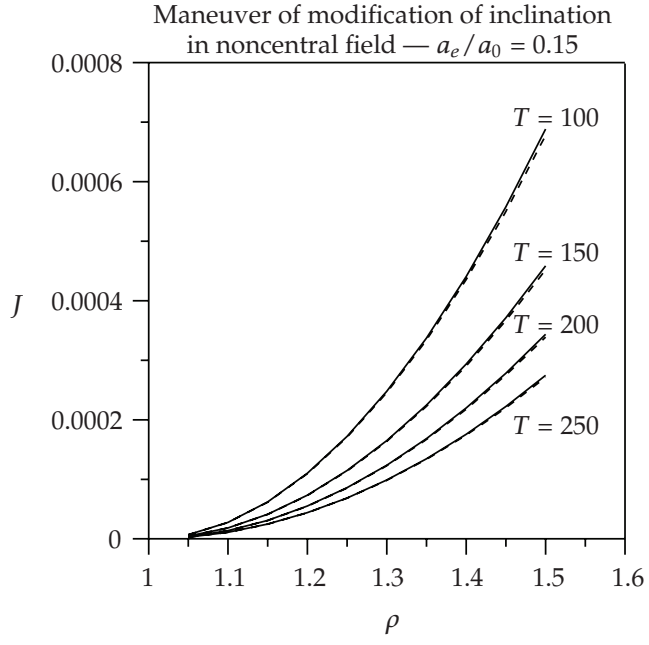

(b)

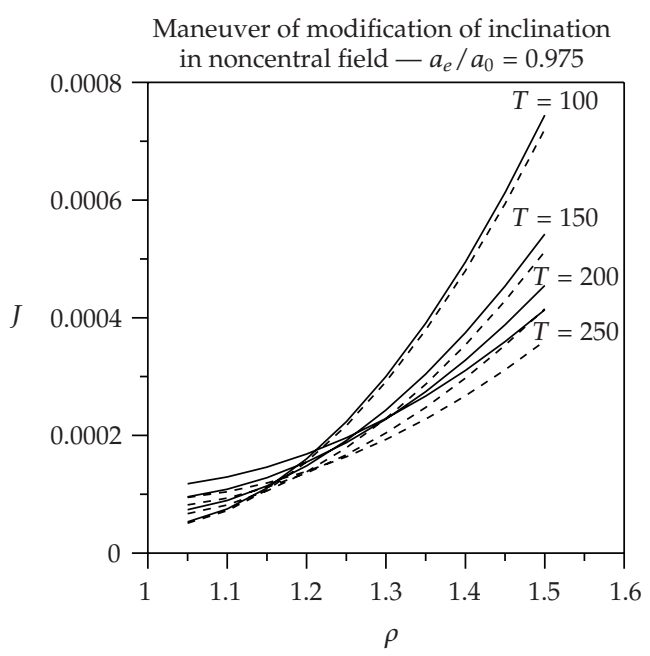

(d)

Figure 5: Consumption for maneuver of modification of inclination.

the initial orbit. Variables $I$ and $\Omega$ are transformed into nonsingular variables $P$ and $Q$ to compare the analytical results with the numerical results. Numerical solutions are obtained by solving the two-point boundary value problem described by (6.4) and (6.5) through a shooting method [34].

Considering the maneuvers described in Tables 1 and 2, Figures 3-5 show that analytical and numerical solutions yield quite similar values of the consumption $J$ in the following cases: (i) maneuvers of modification of all orbital elements with ratio $a_{e} / a_{0}<0.550$; (ii) maneuvers of modification of semimajor axis with ratios $\rho<1.25$ and $a_{e} / a_{0}<0.550$; (iii) maneuvers of modification of inclination with ratios $\rho<1.50$ and $a_{e} / a_{0}<0.550$, for all values of $T$. For maneuvers with very high ratio $a_{e} / a_{0}$, analytical and numerical solutions only yield similar results for the same maneuvers described above for $T=100$ (time units). 


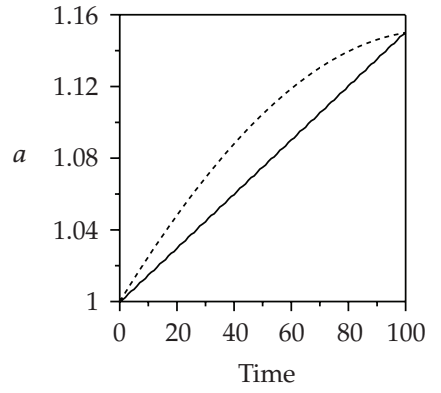

(a)

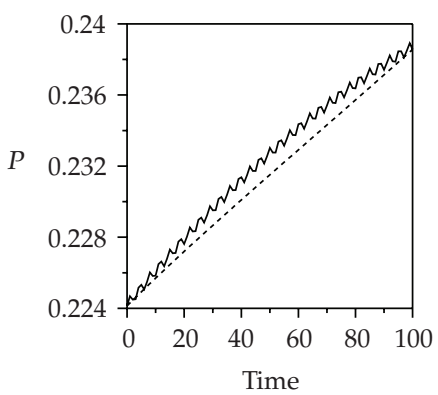

(d)

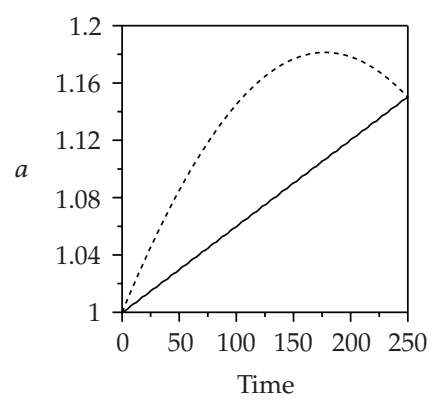

(g)

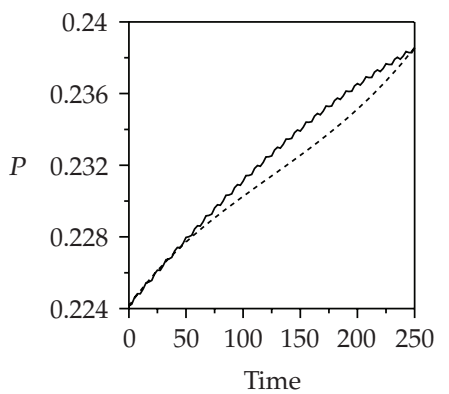

(j)

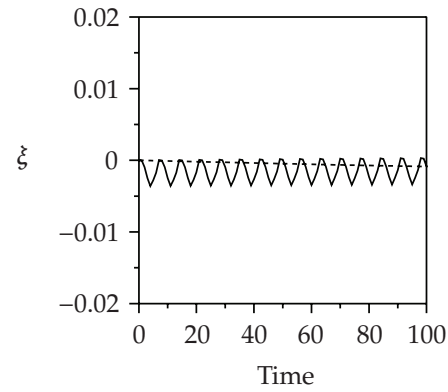

(b)

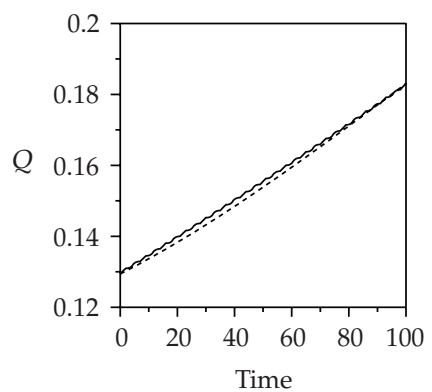

(e)

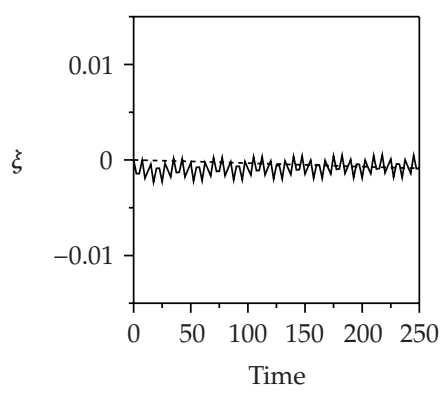

(h)

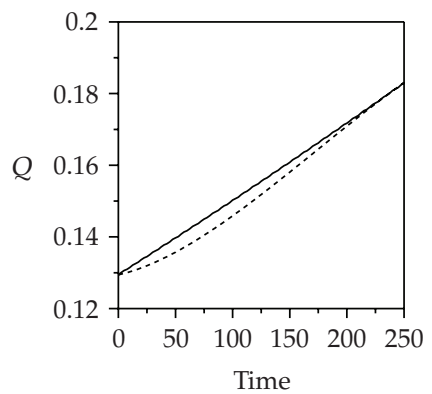

(k)

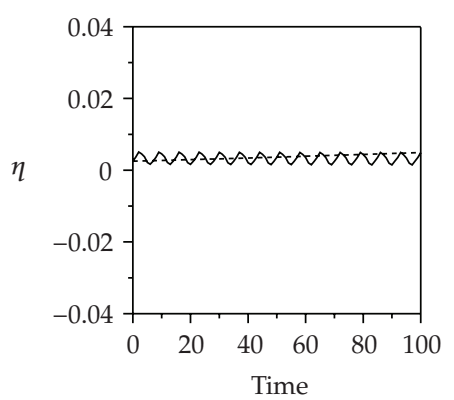

(c)

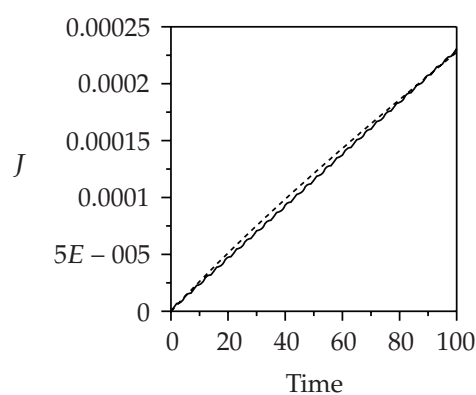

(f)

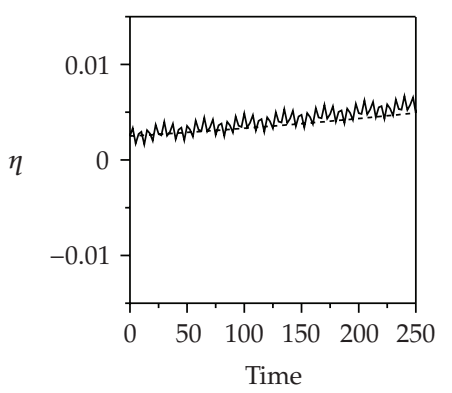

(i)

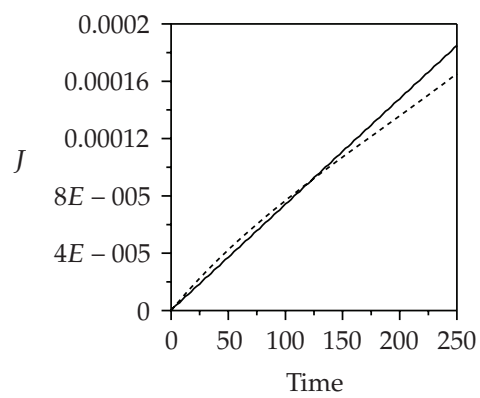

(1)

Figure 6: Time history of state variables for maneuver of modification of all orbital elements with $a_{e} / a_{0}=$ $0.950-T=100$ and 250 . 


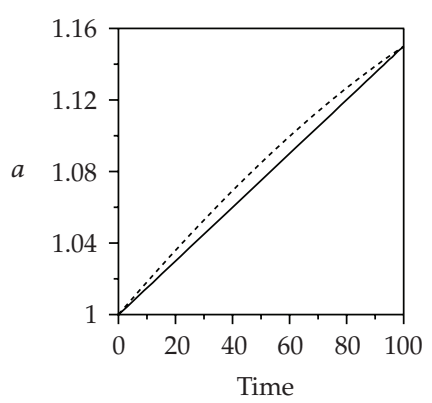

(a)

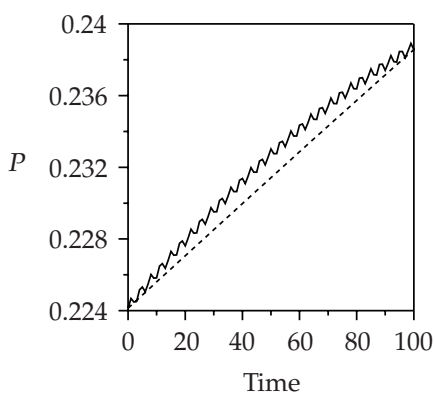

(d)

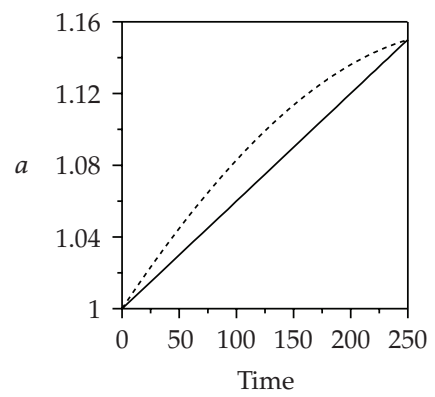

(g)

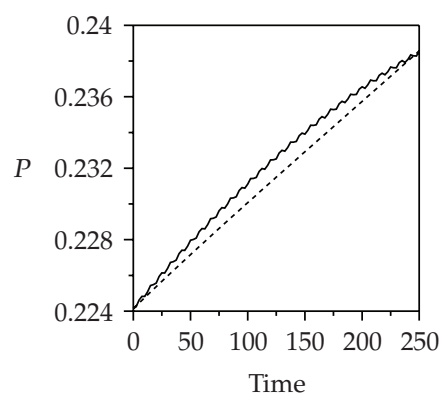

(j)

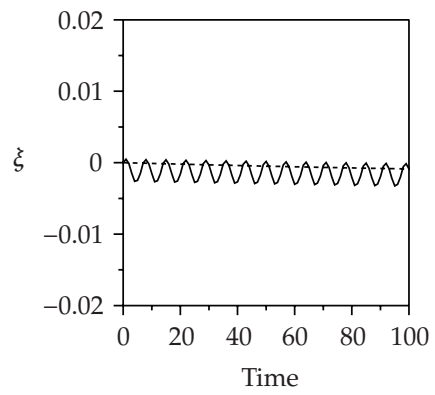

(b)

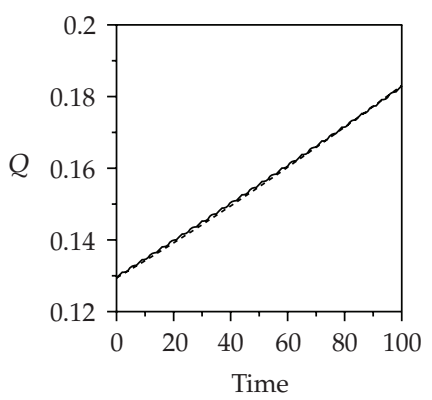

(e)

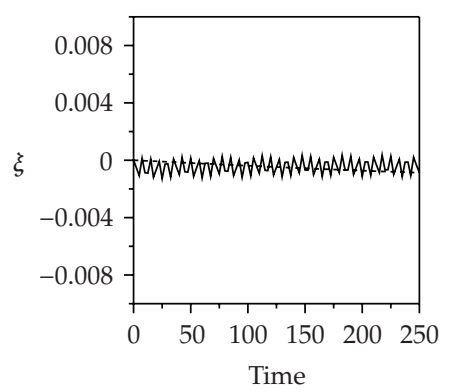

(h)

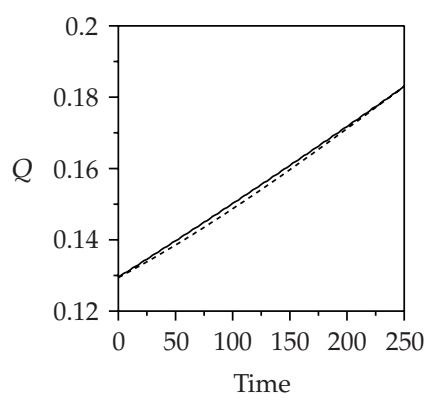

(k)

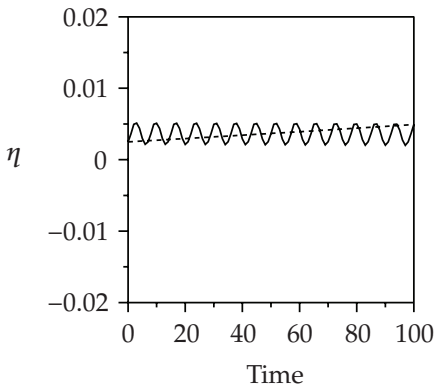

(c)

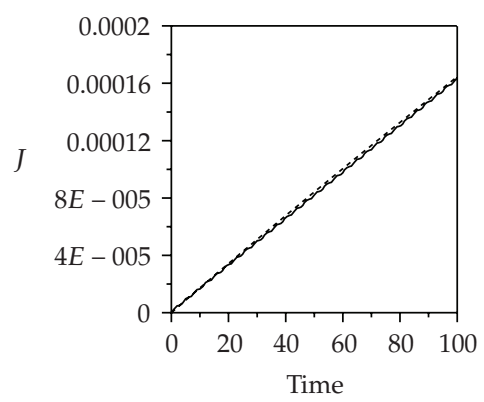

(f)

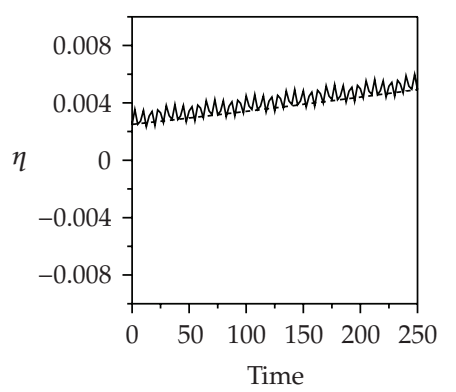

(i)

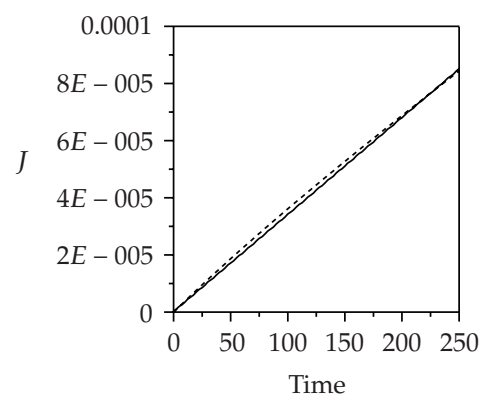

(1)

Figure 7: Time history of state variables for maneuver of modification of all orbital elements with $a_{e} / a_{0}=$ $0.550-T=100$ and 250 . 


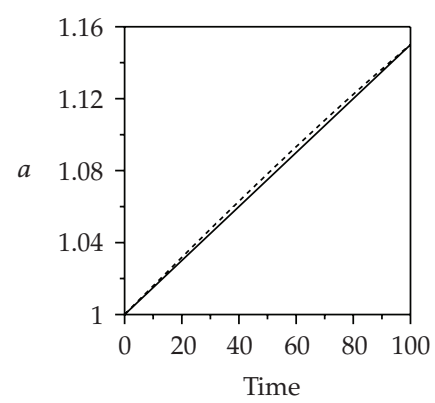

(a)

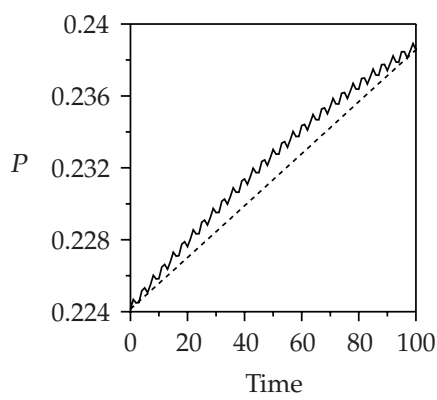

(d)

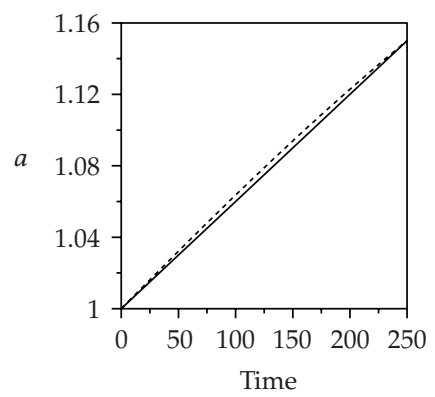

(g)

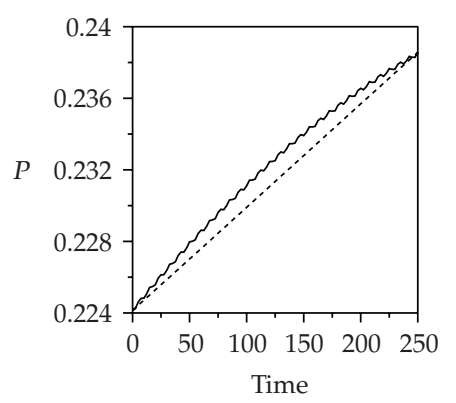

(j)

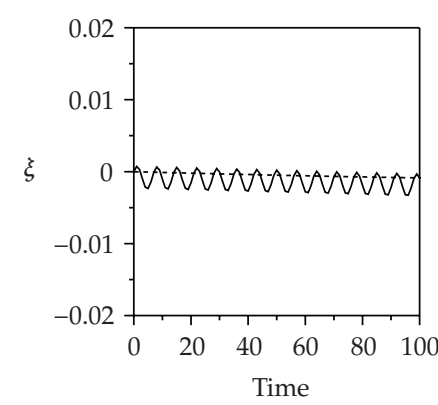

(b)

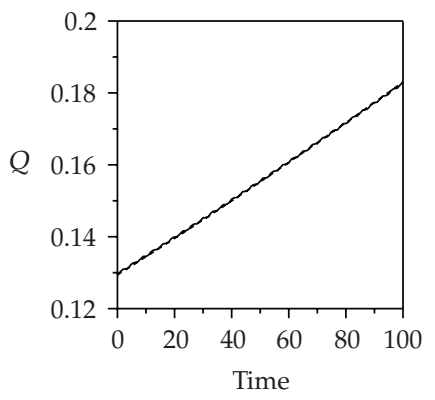

(e)

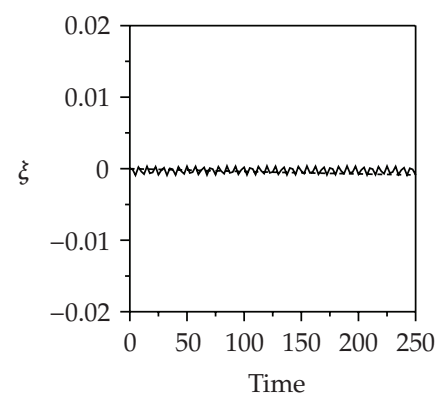

(h)

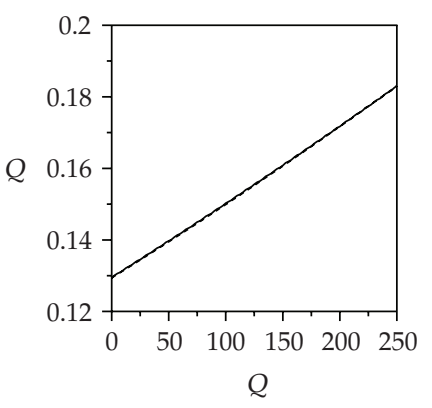

(k)

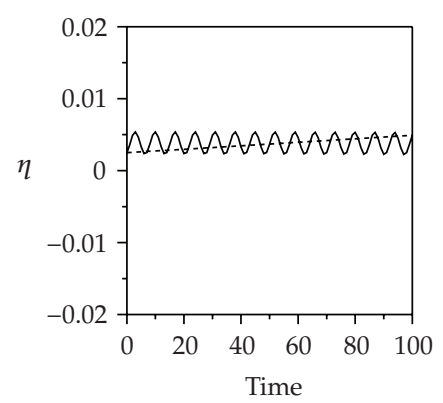

(c)

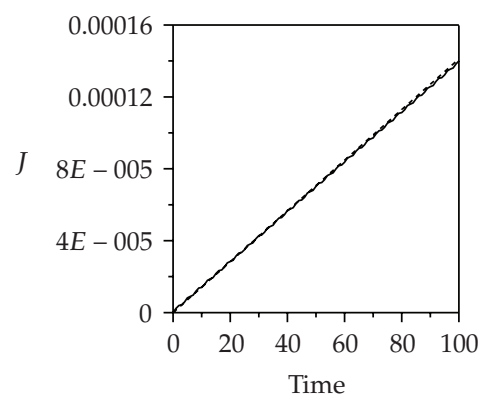

(f)

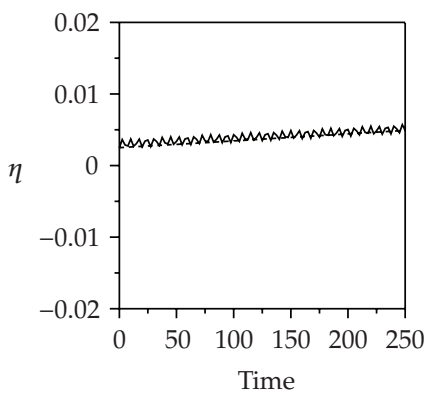

(i)

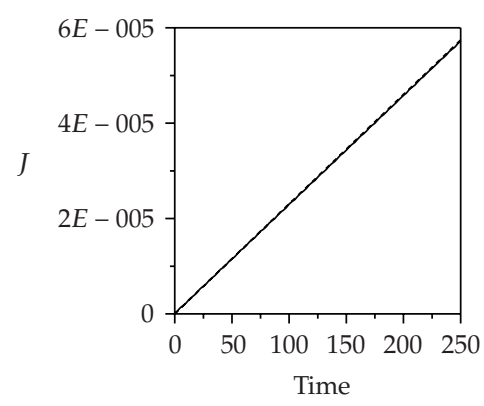

(1)

Figure 8: Time history of state variables for maneuver of modification of all orbital elements with $a_{e} / a_{0}=$ $0.150-T=100$ and 250 . 


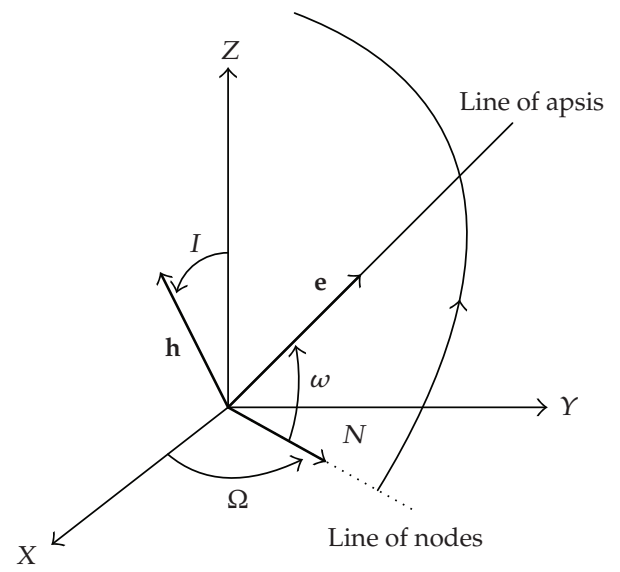

Figure 9: Vectors $\mathbf{e}, \mathbf{h}$, and $\mathbf{N}$.

Figures 6-8 represent the time history of state variables (nonsingular orbital elements) for maneuvers of modification of all orbital elements (Table 1) considering three values of ratios $a_{e} / a_{0}-0.150,0.550$, and 0.950 - and two values of transfer duration $T-100$ and 250 (time units). According to these figures, the analytical solution yields a good representation of the numerical solution for all state variables, excepting the semimajor axis for the maneuver with $a_{e} / a_{0}=0.950$ and $T=250$. The numerical solution shows that the semimajor axis is strongly perturbed by the oblateness of earth, and its time evolution cannot be described by the linear approximation given by the analytical solution. On the other hand, the analytical solutions shows that the amplitude of the short periodic terms decreases as the transfer duration increases such that these terms can be omitted for very long-time transfers.

\section{Conclusion}

In this paper an approach based on canonical transformations is presented for the problem of optimal low-thrust limited-power maneuvers between close orbits with small eccentricities in noncentral gravity which includes the perturbative effects of the second zonal harmonic of the gravitational potential. Analytical first-order solutions are derived through this approach for transfers between nonequatorial orbits and for transfers between near-equatorial orbits. These analytical solutions have been compared to a numerical solution for long-time transfers between arbitrary orbits described by averaged maximum Hamiltonian, expressed in nonsingular orbital elements. The results show that the analytical solutions yield good estimates of the fuel consumption for preliminary mission analysis considering maneuvers with moderate amplitude, ratio $a_{e} / a_{0}$ and transfers duration. Numerical results also show that the semimajor axis is strongly perturbed by the oblateness of earth, and its time evolution cannot be described by the linear approximation given by the analytical solution.

\section{Appendix}

The general solution of the differential equations for the adjoint variables $\mathbf{p}_{r}$ and $\mathbf{p}_{v}$ is obtained by computing the inverse of the Jacobian matrix of the point transformation between 
the Cartesian elements and the orbital ones, defined by (3.2) and (3.3). This matrix is obtained through the variations of the orbital elements induced by the variations in the Cartesian elements, as described below.

Let us consider the inverse of the point transformation defined by (3.2) and (3.3):

$$
\begin{gathered}
a=\frac{r}{2-\left(r v^{2} / \mu\right)}, \\
e^{2}=1-\frac{h^{2}}{\mu a}, \\
\cos I=\frac{\mathbf{k} \bullet \mathbf{h}}{h}, \\
\cos \Omega=\frac{\mathbf{i} \bullet \mathbf{N}}{N}, \\
\cos \omega=\frac{\mathbf{N} \bullet \mathbf{e}}{N e}, \\
\cos E=\frac{1}{e}\left(1-\frac{r}{a}\right),
\end{gathered}
$$

where the eccentricity vector $\mathbf{e}$, the angular momentum vector $\mathbf{h}$, and the nodal vector $\mathbf{N}$, shown in Figure 9, are given, as function of the Cartesian elements $\mathbf{r}$ and $\mathbf{v}$, by the following equations:

$$
\begin{gathered}
\mathbf{e}=\frac{1}{\mu}\left[\left(v^{2}-\frac{\mu}{r}\right) \mathbf{r}-(\mathbf{r} \bullet \mathbf{v}) \mathbf{v}\right], \\
\mathbf{h}=\mathbf{r} \times \mathbf{v}, \\
\mathbf{N}=\mathbf{k} \times \mathbf{h} .
\end{gathered}
$$

Here the symbol $\times$ denotes the cross product. Note that the true anomaly $f$ has been replaced by eccentric anomaly $E$. These anomalies are related through the following equation:

$$
\tan \frac{f}{2}=\sqrt{\frac{1+e}{1-e}} \tan \frac{E}{2} .
$$

Now, consider the variations in the Cartesian elements, $\delta \mathbf{r}$ and $\delta \mathbf{v}$, given in the moving frame of reference by

$$
\begin{gathered}
\delta \mathbf{r}=\delta \xi \mathbf{e}_{\mathbf{r}}+\delta \eta \mathbf{e}_{s}+\delta \zeta \mathbf{e}_{w} \\
\delta \mathbf{v}=\delta u \mathbf{e}_{\mathbf{r}}+\delta v \mathbf{e}_{s}+\delta w \mathbf{e}_{w}
\end{gathered}
$$


The variations of the orbital elements- $a, e, I, \Omega, \omega$, and $E$-induced by the variations in the Cartesian elements, $\delta \mathbf{r}$ and $\delta \mathbf{v}$, are obtained straightforwardly from (A.1) through (A.6) and are given by

$$
\begin{gathered}
\delta a=2\left(\frac{a}{r}\right)^{2} \frac{\mathbf{r} \bullet \delta \mathbf{r}}{r}+\frac{2 a^{2}}{\mu} \mathbf{v} \bullet \delta \mathbf{v}, \\
\delta e=-\frac{\mathbf{h} \bullet \delta \mathbf{h}}{\mu a e}+\frac{h^{2}}{2 \mu a^{2} e} \delta a, \\
\delta I=\frac{\mathbf{h} \bullet \delta \mathbf{h}}{h^{2}} \cot I-\frac{\mathbf{k} \bullet \delta \mathbf{h}}{h} \csc I, \\
\delta \Omega=\frac{\mathbf{N} \bullet \delta \mathbf{N}}{N^{2}} \cot \Omega-\frac{\mathbf{i} \bullet \delta \mathbf{N}}{N} \csc \Omega \\
\delta \omega=\left[\frac{\delta e}{e}+\frac{\mathbf{N} \bullet \delta \mathbf{N}}{N^{2}}\right] \cot \omega-\frac{1}{N e}[\mathbf{N} \bullet \delta \mathbf{e}+\mathbf{e} \bullet \delta \mathbf{N}] \csc \omega, \\
\delta E=\frac{1}{e \sin E}\left[\frac{\mathbf{r} \bullet \delta \mathbf{r}}{r a}-\frac{r}{a^{2}} \delta a+\cos E \delta e\right],
\end{gathered}
$$

where the variations of the vectors $\mathbf{e}, \mathbf{h}$, and $\mathbf{N}$ are written as

$$
\begin{aligned}
\mu \delta \mathbf{e}= & \left(v_{s}^{2} \delta \xi-v_{s} v_{r} \delta \eta+2 r v_{s} \delta v\right) \mathbf{e}_{\mathbf{r}} \\
& +\left(-v_{s} v_{r} \delta \xi+\left(v_{r}^{2}-\frac{\mu}{r}\right) \delta \eta-r v_{s} \delta u-r v_{r} \delta v\right) \mathbf{e}_{s}+\left(\left(v^{2}-\frac{\mu}{r}\right) \delta \zeta-r v_{r} \delta w\right) \mathbf{e}_{w} \\
\delta \mathbf{h}= & -v_{s} \delta \zeta \mathbf{e}_{r}+\left(v_{r} \delta \zeta-r \delta w\right) \mathbf{e}_{s}+\left(v_{s} \delta \xi-v_{r} \delta \eta+r \delta v\right) \mathbf{e}_{\mathbf{w}} \\
\delta \mathbf{N}= & \left(\left(v_{s} \delta \xi-v_{r} \delta \eta+r \delta v\right) \sin I \cos (\omega+f)-\left(v_{r} \delta \zeta-r \delta w\right) \cos I\right) \mathbf{e}_{\mathbf{r}} \\
& +\left(-\left(v_{s} \delta \xi-v_{r} \delta \eta+r \delta v\right) \sin I \sin (\omega+f)-v_{s} \cos I \delta \zeta\right) \mathbf{e}_{s} \\
& +\left(\left(v_{r} \delta \zeta-r \delta w\right) \sin I \sin (\omega+f)+v_{s} \sin I \cos (\omega+f) \delta \zeta\right) \mathbf{e}_{w}
\end{aligned}
$$

Here $v_{r}$ and $v_{s}$ denote the radial and circumferential components of the velocity vector, respectively (see (3.3)). 
In the moving fame of reference, $\mathbf{e}, \mathbf{h}, \mathbf{N}$, and the unit vectors $\mathbf{i}$ and $\mathbf{k}$ are written as

$$
\begin{aligned}
\mathbf{e}= & \left(\frac{h v_{s}}{\mu}-1\right) \mathbf{e}_{\mathbf{r}}-\frac{h v_{r}}{\mu} \mathbf{e}_{s}, \\
\mathbf{h}= & h \mathbf{e}_{\mathbf{w}}=\sqrt{\mu a\left(1-e^{2}\right)} \mathbf{e}_{\mathbf{w}}, \\
\mathbf{N}= & h \sin I \cos (\omega+f) \mathbf{e}_{\mathbf{r}}-h \sin I \sin (\omega+f) \mathbf{e}_{s}, \\
\mathbf{i}= & (\cos \Omega \cos (\omega+f)-\sin \Omega \sin (\omega+f) \cos I) \mathbf{e}_{r} \\
& -(\cos \Omega \sin (\omega+f)+\sin \Omega \cos (\omega+f) \cos I) \mathbf{e}_{s}+\sin \Omega \sin I \mathbf{e}_{w}, \\
\mathbf{k}= & \sin (\omega+f) \sin I \mathbf{e}_{r}+\cos (\omega+f) \sin I \mathbf{e}_{s}+\cos I \mathbf{e}_{w} .
\end{aligned}
$$

From (3.2), (3.3), and (A.2) through (A.11), one gets the explicit form of the variations of the orbital elements- $a, e, I, \Omega, \omega$, and $E$-induced by the variations in the Cartesian elements, $\delta \mathbf{r}$ and $\delta \mathbf{v}$. At this point, it is useful to replace the variation in the eccentric anomaly $E$ by the variation in the mean anomaly $M$, obtained from the well-known Kepler's equation

$$
M=E-e \sin E .
$$

This variation is given by

$$
\delta M=\left(\frac{r}{a}\right) \delta E-\sin E \delta e .
$$

Thus,

$$
\begin{aligned}
& \delta a=2\left(\frac{a}{r}\right)^{2} \delta \xi+\frac{2 e \sin f}{n \sqrt{1-e^{2}}} \delta u+\frac{2 \sqrt{1-e^{2}}}{n}\left(\frac{a}{r}\right) \delta v, \\
& \delta e=\frac{a\left(1-e^{2}\right)}{r^{2}} \cos E \delta \xi+\frac{\sin f}{a} \delta \eta+\frac{\sqrt{1-e^{2}}}{n a} \sin f \delta u+\frac{\sqrt{1-e^{2}}}{n a}(\cos E+\cos f) \delta v, \\
& \delta I=\frac{1}{r}\left(\cos E \sin \omega+\frac{\sin E \cos \omega}{\sqrt{1-e^{2}}}\right) \delta \zeta+\frac{1}{n a \sqrt{1-e^{2}}}\left(\frac{r}{a}\right) \cos (\omega+f) \delta w, \\
& \delta \Omega=\frac{1}{r \sin I}\left(-\cos E \cos \omega+\frac{\sin E \sin \omega}{\sqrt{1-e^{2}}}\right) \delta \zeta+\frac{1}{n a \sin I \sqrt{1-e^{2}}}\left(\frac{r}{a}\right) \sin (\omega+f) \delta w,
\end{aligned}
$$




$$
\begin{aligned}
\delta \omega= & \frac{\sin f}{e r} \delta \xi-\frac{e+\cos f}{a e\left(1-e^{2}\right)} \delta \eta+\frac{\cot I}{r}\left(\cos E \cos \omega-\frac{\sin E \sin \omega}{\sqrt{1-e^{2}}}\right) \delta \zeta \\
& -\frac{\sqrt{1-e^{2}}}{n a e} \cos f \delta u+\frac{\sqrt{1-e^{2}}}{n a e} \sin f\left(1+\frac{1}{1+e \cos f}\right) \delta v \\
& -\frac{\cot I}{n a \sqrt{1-e^{2}}}\left(\frac{r}{a}\right) \sin (\omega+f) \delta w, \\
\delta M= & -\frac{1-e^{3} \cos E}{e r \sqrt{1-e^{2}}} \sin f \delta \xi+\frac{\sqrt{1-e^{2}}}{a e} \cos f \delta \eta \\
& +\frac{\left(1-e^{2}\right)}{n a e}\left(\cos f-\frac{2 e}{1+e \cos f}\right) \delta u-\frac{\left(1-e^{2}\right)}{n a e} \sin f\left(1+\frac{1}{1+e \cos f}\right) \delta v .
\end{aligned}
$$

Equations (A.16) can be put in the form

$$
\left[\begin{array}{l}
\delta a \\
\delta e \\
\delta I \\
\delta \Omega \\
\delta \omega \\
\delta M
\end{array}\right]=\Delta^{-1}\left[\begin{array}{l}
\delta \xi \\
\delta \eta \\
\delta \zeta \\
\delta u \\
\delta v \\
\delta w
\end{array}\right]
$$

where the matrix $\Delta^{-1}$ is inverse Jacobian matrix of the point transformation between the Cartesian elements and the orbital ones, defined by (3.2) and (3.3).

Following the properties of generalized canonical systems [32], the general solution of the differential equations for the adjoint variables $\mathbf{p}_{r}$ and $\mathbf{p}_{v}$ is given by

$$
\left[\begin{array}{l}
\mathbf{p}_{\mathbf{r}} \\
\mathbf{p}_{\mathbf{v}}
\end{array}\right]=\left(\Delta^{-1}\right)^{T}\left[\begin{array}{c}
p_{a} \\
p_{e} \\
p_{I} \\
p_{\Omega} \\
p_{\omega} \\
p_{M}
\end{array}\right]
$$

with $\mathbf{p}_{r}$ and $\mathbf{p}_{v}$ expressed in the moving frame of reference. Equations (3.4) and (3.5) are obtained straightforwardly from the above equation.

\section{Acknowledgment}

This research has been supported by CNPq under contract 305049/2006-2. 


\section{References}

[1] T. N. Edelbaum, "Optimum low-thrust rendezvous and station keeping," AIAA Journal, vol. 2, no. 7, pp. 1196-1201, 1964.

[2] T. N. Edelbaum, "Optimum power-limited orbit transfer in strong gravity fields," AIAA Journal, vol. 3, no. 5, pp. 921-925, 1965.

[3] J.-P. Marec, Transferts Optimaux Entre Orbites Elliptiques Proches, ONERA Publication no. 121, ONERA, Châtillon, France, 1967.

[4] J.-P. Marec and N. X. Vinh, “Optimal low-thrust, limited power transfers between arbitrary elliptical orbits," Acta Astronautica, vol. 4, no. 5-6, pp. 511-540, 1977.

[5] J.-P. Marec, Optimal Space Trajectories, Elsevier Scientific, New York, NY, USA, 1979.

[6] S. da Silva Fernandes, "Optimal low-thrust transfer between neighbouring quasi-circular orbits around an oblate planet," Acta Astronautica, vol. 19, no. 12, pp. 933-938, 1989.

[7] S. da Silva Fernandes and W. Sessin, "Optimal low-thrust limited power transfer between neighbouring quasi-circular orbits of small inclinations around an oblate planet," Acta Astronautica, vol. 19, no. 5, pp. 401-409, 1989.

[8] S. da Silva Fernandes and W. Sessin, "Long-time, low-thrust, limited power transfer between neighboring quasi-circular orbits in a noncentral force field," in Proceedings of the International Symposium on Space Dynamics, pp. 823-831, Cepadues-Editions, Toulouse, France, November 1990.

[9] C. M. Haissig, K. D. Mease, and N. X. Vinh, "Minimum-fuel, power-limited transfers between coplanar elliptical orbits," Acta Astronautica, vol. 29, no. 1, pp. 1-15, 1993.

[10] S. da Silva Fernandes, "Optimum low-thrust manoeuvres in a noncentral gravity field," Advances in the Astronautical Sciences, vol. 84, part 2, pp. 1334-1349, 1993.

[11] V. Coverstone-Carroll and S. N. Williams, "Optimal low thrust trajectories using differential inclusion concepts," The Journal of the Astronautical Sciences, vol. 42, no. 4, pp. 379-393, 1994.

[12] J. A. Kechichian, "Optimal low-thrust transfer using variable bounded thrust," Acta Astronautica, vol. 36, no. 7, pp. 357-365, 1995.

[13] J. A. Kechichian, "Optimal low-thrust rendezvous using equinoctial orbit elements," Acta Astronautica, vol. 38, no. 1, pp. 1-14, 1996.

[14] J. A. Kechichian, "Trajectory optimization using nonsingular orbital elements and true longitude," Journal of Guidance, Control, and Dynamics, vol. 20, no. 5, pp. 1003-1009, 1997.

[15] S. Geffroy and R. Epenoy, "Optimal low-thrust transfers with constraints-generalization of averaging techniques," Acta Astronautica, vol. 41, no. 3, pp. 133-149, 1997.

[16] J. A. Kechichian, "Reformulation of Edelbaum's low-thrust transfer problem using optimal control theory," Journal of Guidance, Control, and Dynamics, vol. 20, no. 5, pp. 988-994, 1997.

[17] C. A. Kluever and S. R. Oleson, "A direct approach for computing near-optimal low-thrust transfers," in AAS/AIAA Astrodynamics Specialist Conference, Sun Valley, Idaho, USA, August 1997, AAS paper 97 717.

[18] V. Coverstone-Carroll, J. W. Hartmann, and W. J. Mason, "Optimal multi-objective low-thrust spacecraft trajectories," Computer Methods in Applied Mechanics and Engineering, vol. 186, no. 2-4, pp. 387-402, 2000.

[19] M. Vasile, F. B. Zazzera, R. Jehn, and G. Janin, “Optimal interplanetary trajectories using a combination of low-thrust and gravity assist manoeuvres," in Proceedings of the 51th IAF International Astronautical Congress, Rio de Janeiro, Brazil, October 2000, IAF-00-A.5.07.

[20] A. A. Sukhanov, "Optimization of low-thrust interplanetary transfers," Cosmic Research, vol. 38, no. 6, pp. 584-587, 2000.

[21] A. A. Sukhanov and A. F. B. de A. Prado, "Constant tangential low-thrust trajectories near on oblate planet," Journal of Guidance, Control, and Dynamics, vol. 24, no. 4, pp. 723-731, 2001.

[22] R. Bertrand, J. Bernussou, S. Geffroy, and R. Epenoy, "Electric transfer optimization for Mars sample return mission," Acta Astronautica, vol. 48, no. 5-12, pp. 651-660, 2001.

[23] B. M. Kiforenko, Z. V. Pasechnik, S. B. Kyrychenko, and I. Y. Vasiliev, "Minimum time transfers of a low-thrust rocket in strong gravity fields," Acta Astronautica, vol. 52, no. 8, pp. 601-611, 2003.

[24] G. D. Racca, "New challenges to trajectory design by the use of electric propulsion and other new means of wandering in the solar system," Celestial Mechanics E Dynamical Astronomy, vol. 85, no. 1, pp. 1-24, 2003. 
[25] B. N. Kiforenko, "Optimal low-thrust transfers between orbits in a central gravitational field," International Applied Mechanics, vol. 41, no. 11, pp. 1211-1238, 2005.

[26] B. Bonnard, J.-B. Caillau, and R. Dujol, "Averaging and optimal control of elliptic Keplerian orbits with low propulsion," Systems E Control Letters, vol. 55, no. 9, pp. 755-760, 2006.

[27] J.-P. Marec, "Trajectoires spatiales optimales," in Space Mathematics, pp. 77-95, Cépaduès, Toulouse, France, 1984.

[28] L. S. Pontryagin, V. G. Boltyanskii, R. V. Gamkrelidze, and E. F. Mishchenko, The Mathematical Theory of Optimal Processes, John Wiley \& Sons, New York, NY, USA, 1962.

[29] G. Hori, "Theory of general perturbations with unspecified canonical variables," Publications of the Astronomical Society of Japan, vol. 18, no. 4, p. 287, 1966.

[30] W. M. Kaula, Theory of Satellite Geodesy, Blaisdell, Waltham, Mass, USA, 1966.

[31] R. H. Battin, An Introduction to the Mathematics and Methods of Astrodynamics, AIAA Education Series, American Institute of Aeronautics and Astronautics, Washington, DC, USA, 1987.

[32] S. da Silva Fernandes, "Generalized canonical systems-I: general properties," Acta Astronautica, vol. 32, no. 5, pp. 331-338, 1994.

[33] C. Lánczos, The Variational Principles of Mechanics, Dover, New York, NY, USA, 4th edition, 1970.

[34] J. Stoer and R. Bulirsch, Introduction to Numerical Analysis, vol. 12 of Texts in Applied Mathematics, Springer, New York, NY, USA, 3rd edition, 2002. 


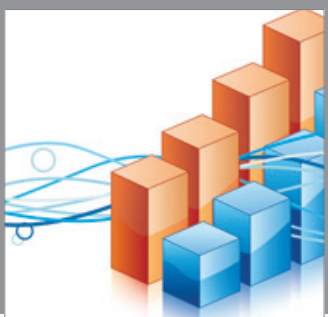

Advances in

Operations Research

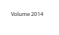

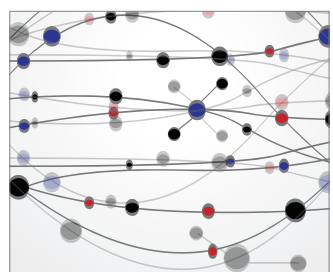

\section{The Scientific} World Journal
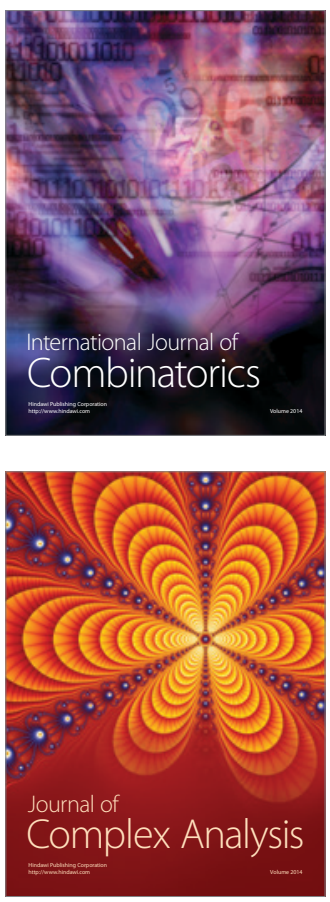

International Journal of

Mathematics and

Mathematical

Sciences
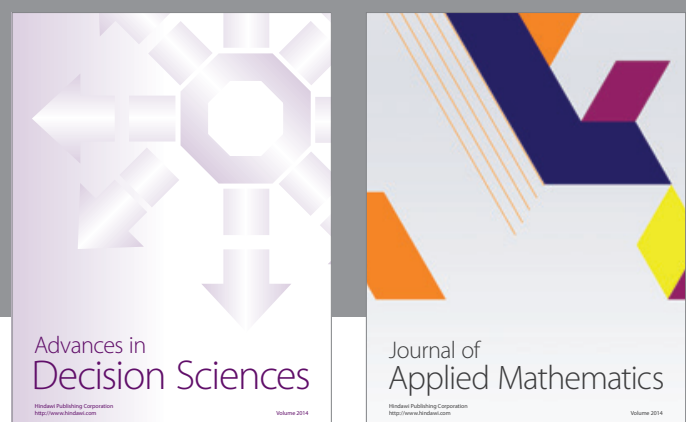

Journal of

Applied Mathematics
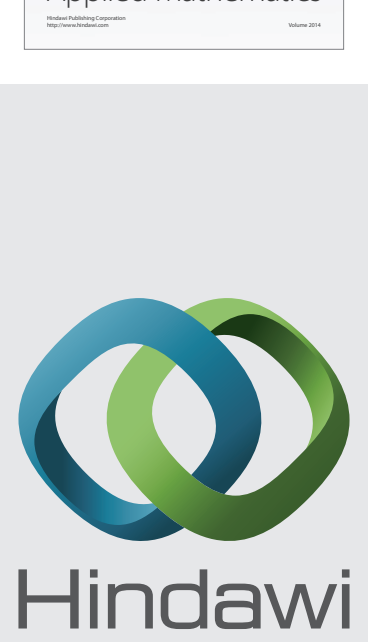

Submit your manuscripts at http://www.hindawi.com
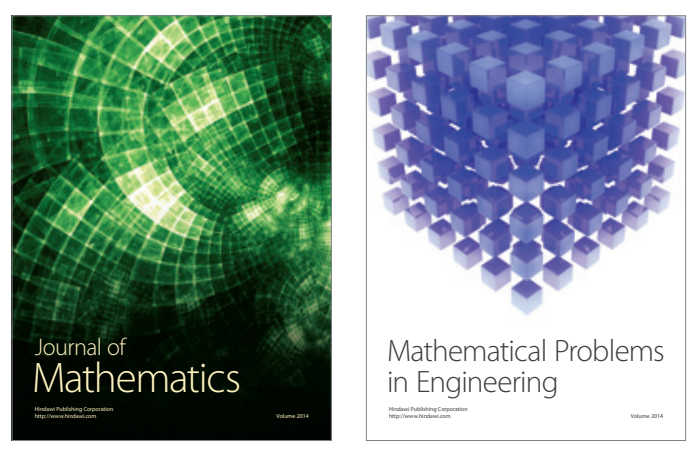

Mathematical Problems in Engineering
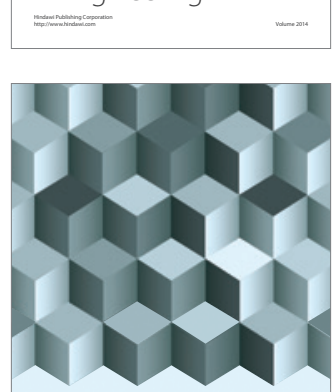

Journal of

Function Spaces
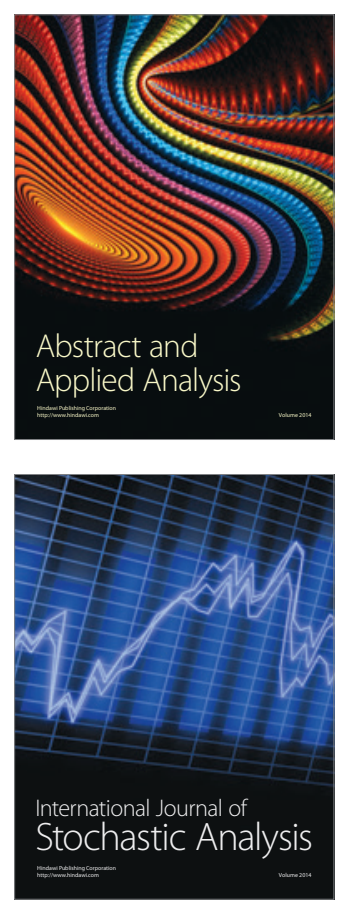

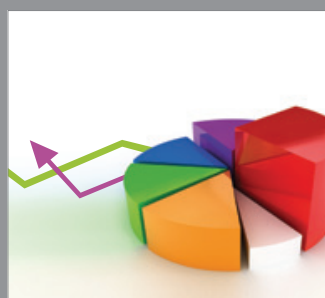

ournal of

Probability and Statistics

Promensencen
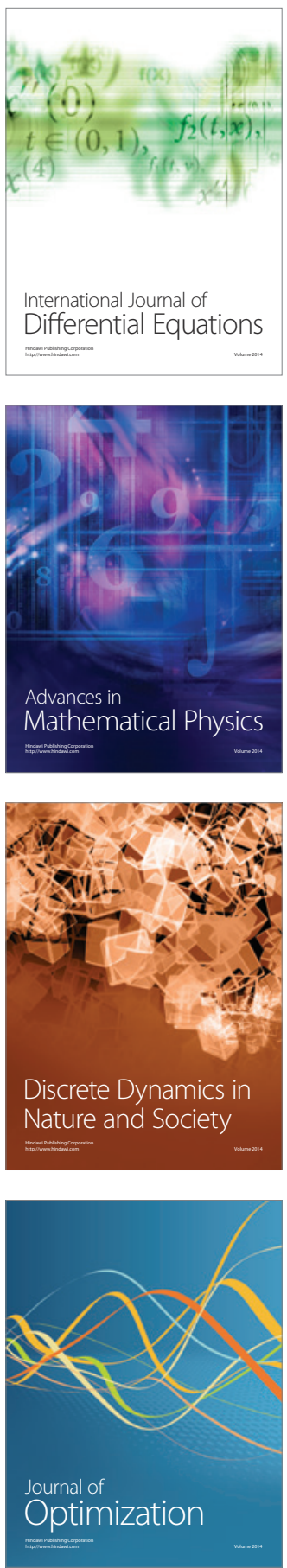